PACS: 05.60.+w, 05.70.Ln, 05.20.Dd, 52.25.Dg, 52.25.Fi

\title{
NONSTATIONARY SOLUTION TO THE ENSKOG-LANDAU KINETIC EQUATION USING BOUNDARY CONDITIONS METHOD
}

\author{
A.E.Kobryn, I.P.OmELyan, M.V.TOKARChUK \\ Institute for Condensed Matter Physics \\ Ukrainian National Academy of Sciences, \\ 1 Svientsitskii St., UA-290011 Lviv-11, Ukraine
}

Received January 7, 1996

\begin{abstract}
Nonstationary and nonequilibrium process is considered on the basis of the Enskog-Landau kinetic equation using boundary conditions method. A nonstationary solution to this equation is found in the pair collision approximation. The influence of the long-range interparticle correlations are taken into account sequentially for the first time. Transport coefficients with additional new terms are calculated. Limiting cases of the stationary process, low densities are considered. An application of the boundary conditions method to hydrodynamic description of fast processes is discussed.
\end{abstract}

\section{Introduction}

The development of methods for construction of the nonequilibrium processes theory in dense gases, liquids and plasmas is an important direction in the modern theoretical physics. Moreover the construction of kinetic equations for such classical and quantum systems still remains to be a major problem in the kinetic theory. It is complicated additionally in the case of dense gases, liquids and plasmas where kinetics and hydrodynamics are closely related and should be considered simultaneously [1-5].

An approach for construction of kinetic equations from the first principles of statistical mechanics, namely from the Liouville equation, has been developed in [6-10]. It is based on the principle of weakening of correlations between particles in time. In the framework of this approach, the Boltzmann kinetic equation, which satisfactorily describes nonequilibrium properties and transport processes in dilute systems, can be obtained easily as a zeroth approximation on density. However this approach becomes unusable when the density increases and can not be a small parameter of the theory. One way to obtain appropriate description of a dense system lies in using Enskog theory SET (Standard Enskog Theory) $[11,12]$. The Enskog equation plays an appreciable part in the kinetic theory next to the Boltzmann one [13]. This equation has been obtained at modelling of molecules by hard spheres for dense gases. As a result the collision integral has been presented in the analytical form. It is reached by advantage of hard sphere model, where collisions can be considered as momentary ones and by the fact that multiparticle contact at the same time is reputed to be infinitely small. Density correction, introduced by Enskog, proved to be considerable as far as transport, due to collisions in a dense system, is the main mechanism of transport. Each molecule is almost localized in one point of the space by surrounding neighbouring molecules and therefore flow transport is suppressed. Though this theory describes density dependence of kinetic coefficients appropriately, at the same time the suppositions as to the structure of collision integral remain sufficiently rough and phenomenological.

(C) A.E.Kobryn, I.P.Omelyan, M.V.Tokarchuk, 1996

ISSN 0452-9910. Condensed Matter Physics 1996 No 8 (75-98) 
Next affirmative steps in developing of kinetic theory for dense systems have been made on the basis of SET modified MET (Modified Enskog Theory) and revised RET (Revised Enskog Theory) theories for dense gases [14] and generalization of them to the case of presence of some long-range interaction KMFT (Kinetic Mean Field Theory) [15,16]. So-called DRS kinetic theory (Davis-Rice-Sangers) has been proposed in [17]. Interparticle interaction potential is considered here as a finite depth square well. Revised variant of this theory RDRS (Revised DRS) has been realized in [18].

An approach for obtaining kinetic equations for dense systems, which is based on ideas of papers [6,10], has been proposed in [1] and generalized in [2,3]. Here the formulation of modified boundary condition for the BBGKY hierarchy is used with taking into account corrections connected with local conservation laws. It allowed to obtain in the pair collision approximation an Enskog-type kinetic equation without any phenomenological assumptions. Similar ideas with other modification of the approach [6] have been advanced and developed in [19-23] and in monograph [24]. Moreover in [19-24] generalization of the approach to the case of presence of nonsingular interaction potentials has been attempted. The same question was produced and solved partially in [25-28] (within approach of [19-24]) and in [29-32], and in [33] using hydrodynamical methods only.

Generalized version of RDRS - GDRS was considered in [34] being based on approaches of [1]. Here, to treat a more realistic model a kinetic equation for dense classical systems is offered with interparticle interaction potential in the shape of a multiple-step function, where its sequential derivation and the normal solution have been obtained.

Concrete successes in developing kinetic theory for dense gases and liquids stimulated transferring new methods for description of nonequilibrium properties to the systems with long-range interactions, including Coulombic ones as well. First of all it is due to the fact that pure hard sphere model can not be used for predominant of majority systems. Taking into account reality of intermolecular interaction potential is needed. For example, it is partially done in $[29,30,35,36]$. We also note, that the choice of more realistic interaction potentials was also accompanied by the generalization of existing theories to the case of multicomponent liquids and gases [37], in $[38,39]$ to the case of chemically reactive gases as well as by the complication of systems under consideration [40-42]. It is necessary to note that just as on the stage of choicing of long-range interaction potential between particles by Coulombic one, we obtain another type of problems, which demand for their solution, possibly, qualitatively new approaches. In particular it concerns the case when long-range part of an interparticle interaction potential carries electric character (Coulomb one). In fact then one deals not with dense gases but with the dense plasma. Obstacles in considering such systems are well known [43-49]. They consist mostly in ambiguity of collision integral construction, which depends directly on a method of plasma "formation" [50], i.e., taking into consideration strong interactions of particles at short distances (in the pair collision approximation) and collective interaction of particles at long distances that corresponds to the polarization approximation. It is noted in [29,30,51-55] that for studying of transport processes in ionized gases (plasma) the Enskog's method is applicable in general, but inconvenience still remains: collision integrals for a Coulomb system reveal the nonanalytical behaviour at both small and long distances. As was noted in [2], one can get rid of divergency at short-range distances by a certain choice of shortrange interaction potential. But to avoid this problem sequentially at long-range distances, including of dynamic screening effects is needed or taking the long-range interaction potential as a screened one (see for example [56]). Among other difficulties it is necessary to underline the absence of a small parameter on which one can perform an expansion to obtain a kinetic equation. It should be mentioned that such small parameters in the kinetic theory of dilute gases $[45,57,58]$ is reduced density $\varepsilon=n / \sigma^{3}$, and in plasmas theory - plasma parameter $\mu=\frac{Z_{\alpha} Z_{\beta} e^{2}}{k T R_{D}}$, where $R_{D}=\sqrt{\frac{k T}{4 \pi n e^{2}}}$ is the Debye radius. Quantities $\varepsilon$ and $\mu$ characterize the role of 
interaction between particles in a kinetic equation. Dissipative and nondissipative processes depend on this interaction.

In this paper Enskog-Landau kinetic equation for onecomponent system of charged hard spheres, which has been obtained in [2], is considered. Normal solution for the kinetic equation has been found in the first approximation using Chapman-Enskog method $[13,59]$. This solution allows to calculate and write down transport coefficients in an analytical form. A similar approach is applied for a multicomponent system of charged hard spheres and transport coefficients for such system have been found $[60,61]$. The simplicity and evidence of the Chapman-Enskog method for solving kinetic equations enabled to transfer this method rather easy on systems treated in [2]. The systems are to be thought as those with moderate and high density. But this method allows to find the unknown one-particle distribution function as far as transport coefficients in the stationary case only. As it is well known, the Chapman-Enskog method can not come beyond a stationary process. Similar drawbacks are peculiar to the Grad method [62,63], which is oftenly used to solve kinetic equations next to Chapman-Enskog method. In the present paper to find the normal solution to considered kinetic equation, so-called boundary conditions method is used, which has been introduced in [64]. The normal solution to the classical Boltzmann equation for a dilute gas [11-13,44,45], obtained by this method in limiting stationary case, completely coincides with the result derived using other methods: the Chapman-Enskog method [11-13], the Grad method $[62,63]$ and others $[65,66]$. In the next paper [67] a new approach has been developed and applied to finding the normal solution of Boltzmann equation for multicomponent systems.

\section{Enskog-Landau kinetic equation and conservation laws}

\subsection{Modified BBGKY hierarchy}

Enskog-Landau kinetic equation for one-particle distribution function of onecomponent system of charged hard spheres $f_{1}\left(x_{1} ; t\right)$ has been obtained in [2], taking into account a modified limiting condition in the pair collision approximation. First two equations of the hierarchy are $[1,10]$ :

$$
\begin{aligned}
& \left\{\frac{\partial}{\partial t}+\boldsymbol{v}_{1} \frac{\partial}{\partial \boldsymbol{r}_{1}}\right\} f_{1}\left(x_{1} ; t\right)- \\
& \frac{1}{m} \int \mathrm{d} x_{2} \frac{\partial}{\partial \boldsymbol{r}_{1}} \Phi\left(\left|\boldsymbol{r}_{12}\right|\right)\left\{\frac{\partial}{\partial \boldsymbol{v}_{1}}-\frac{\partial}{\partial \boldsymbol{v}_{2}}\right\} f_{2}\left(x_{1}, x_{2} ; t\right)=0, \\
& \left\{\frac{\partial}{\partial t}+\mathrm{i} L_{2}+\varepsilon\right\} f_{2}\left(x_{1}, x_{2} ; t\right)=\varepsilon g_{2}\left(\boldsymbol{r}_{1}, \boldsymbol{r}_{2} ; t\right) f_{1}\left(x_{1} ; t\right) f_{1}\left(x_{2} ; t\right) .
\end{aligned}
$$

Here $\varepsilon \rightarrow 0$ is performed after thermodynamical limiting transition; $\Phi\left(\left|\boldsymbol{r}_{12}\right|\right)$ is the total interparticle interaction potential of charged hard spheres, which consists of short-range $\Phi^{h s}\left(\left|\boldsymbol{r}_{12}\right|\right)$ and long-range $\Phi^{l}\left(\left|\boldsymbol{r}_{12}\right|\right)$ parts; $x \equiv\{\boldsymbol{r}, \boldsymbol{p}\}$ is the aggregate of coordinates and momenta, they are defined for each particle in a $\mathbb{R}^{3}$ space [2], $\boldsymbol{v}=m^{-1} \boldsymbol{p}$ denotes velocity of the particle;

$$
\mathrm{i} L_{2}=\boldsymbol{v}_{1} \frac{\partial}{\partial \boldsymbol{r}_{1}}+\boldsymbol{v}_{2} \frac{\partial}{\partial \boldsymbol{r}_{2}}-\frac{\partial}{\partial \boldsymbol{r}_{1}} \Phi\left(\left|\boldsymbol{r}_{12}\right|\right) \frac{1}{m}\left\{\frac{\partial}{\partial \boldsymbol{v}_{1}}-\frac{\partial}{\partial \boldsymbol{v}_{2}}\right\} \text {. }
$$

$g_{2}\left(\boldsymbol{r}_{1}, \boldsymbol{r}_{2} ; t\right) \equiv g_{2}\left(\boldsymbol{r}_{1}, \boldsymbol{r}_{2} \mid \beta(t), n(t)\right)$ is a quasiequilibrium binary correlation function of a system of charged hard spheres, it being a functional of local particle density $n\left(\boldsymbol{r}_{1} ; t\right)$ and reciprocal temperature analogue $\beta\left(\boldsymbol{r}_{1} ; t\right)$ [1]. Formal solution of equation to $f_{2}\left(x_{1}, x_{2} ; t\right)$ reads:

$$
f_{2}\left(x_{1}, x_{2} ; t\right)=
$$




$$
\varepsilon \int_{-\infty}^{0} \mathrm{~d} \tau \exp \left\{\left(\varepsilon+\mathrm{i} L_{2}\right) \tau\right\} g_{2}\left(\boldsymbol{r}_{1}, \boldsymbol{r}_{2} ; t+\tau\right) f_{1}\left(x_{1} ; t+\tau\right) f_{1}\left(x_{2} ; t+\tau\right)
$$

\subsection{Enskog-Landau kinetic equation}

Substituting the solution for $f_{2}\left(x_{1}, x_{2} ; t\right)$ into equation for $f_{1}\left(x_{1} ; t\right)$ after some approximations [68], one can obtain the Enskog-Landau kinetic equation for onecomponent system of charged hard spheres for a nonequilibrium one-particle distribution function

$$
\left\{\frac{\partial}{\partial t}+\boldsymbol{v}_{1} \frac{\partial}{\partial r_{1}}\right\} f_{1}\left(x_{1} ; t\right)=I_{E}\left(x_{1} ; t\right)+I_{M F}\left(x_{1} ; t\right)+I_{L}\left(x_{1} ; t\right) .
$$

The right-hand side of this equation is the so-called generalized EnskogLandau collision integral, where each term can be considered as a separate collision integral. Their structure are as follows:

$I_{E}\left(x_{1} ; t\right)$ is the collision integral of the Enskog theory RET [2]:

$$
\begin{aligned}
& I_{E}\left(x_{1} ; t\right)=\sigma^{2} \int \mathrm{d} \hat{\boldsymbol{r}}_{12} \mathrm{~d} \boldsymbol{v}_{2} \Theta\left(\hat{\boldsymbol{r}}_{12} \boldsymbol{g}\right)\left(\hat{\boldsymbol{r}}_{12} \boldsymbol{g}\right) \times \\
& \left\{g_{2}\left(\boldsymbol{r}_{1}, \boldsymbol{r}_{1}+\hat{\boldsymbol{r}}_{12} \sigma ; t\right) f_{1}\left(\boldsymbol{r}_{1}, \boldsymbol{v}_{1}^{\prime} ; t\right) f_{1}\left(\boldsymbol{r}_{1}+\hat{\boldsymbol{r}}_{12} \sigma, \boldsymbol{v}_{2}^{\prime} ; t\right)-\right. \\
& \left.g_{2}\left(\boldsymbol{r}_{1}, \boldsymbol{r}_{1}-\hat{\boldsymbol{r}}_{12} \sigma ; t\right) f_{1}\left(\boldsymbol{r}_{1}, \boldsymbol{v}_{1} ; t\right) f_{1}\left(\boldsymbol{r}_{1}-\hat{\boldsymbol{r}}_{12} \sigma, \boldsymbol{v}_{2} ; t\right)\right\},
\end{aligned}
$$

where $\sigma$ is a hard sphere diameter, $\boldsymbol{g}$ denotes a vector of relative velocity for two particles, $\hat{\boldsymbol{r}}_{12}$ is a unit vector along the direction between centres of particles 1 and 2,

$$
\begin{array}{ll}
\boldsymbol{v}_{1}^{\prime}=\boldsymbol{v}_{1}+\hat{\boldsymbol{r}}_{12}\left(\hat{\boldsymbol{r}}_{12} \cdot \boldsymbol{g}\right), & \boldsymbol{g}=\boldsymbol{v}_{2}-\boldsymbol{v}_{1}, \\
\boldsymbol{v}_{2}^{\prime}=\boldsymbol{v}_{2}-\hat{\boldsymbol{r}}_{12}\left(\hat{\boldsymbol{r}}_{12} \cdot \boldsymbol{g}\right), & \hat{\boldsymbol{r}}_{12}=\left|\boldsymbol{r}_{12}\right|^{-1} \boldsymbol{r}_{12} ;
\end{array}
$$

$I_{M F}\left(x_{1} ; t\right)$ is the collision integral of the kinetic mean field theory KMFT $[15,16]$ :

$$
I_{M F}\left(x_{1} ; t\right)=\int \mathrm{d} x_{2} \frac{\partial \Phi^{l}\left(\left|\boldsymbol{r}_{12}\right|\right)}{m \partial \boldsymbol{r}_{1}} \frac{\partial}{\partial \boldsymbol{v}_{1}} g_{2}\left(\boldsymbol{r}_{1}, \boldsymbol{r}_{2} ; t\right) f_{1}\left(x_{1} ; t\right) f_{1}\left(x_{2} ; t\right),(2
$$

where $\Phi^{l}\left(\left|\boldsymbol{r}_{12}\right|\right)$ is a long-range part of an interparticle interaction potential;

$I_{L}\left(x_{1} ; t\right)$ is generalized Landau collision integral [2]:

$$
\begin{aligned}
& I_{L}\left(x_{1} ; t\right)=\frac{1}{m^{2}} \frac{\partial}{\partial \boldsymbol{v}_{1}} \int \mathrm{d} x_{2} g_{2}\left(\boldsymbol{r}_{1}, \boldsymbol{r}_{2} ; t\right)\left[\frac{\partial \Phi^{l}\left(\left|\boldsymbol{r}_{12}\right|\right)}{\partial \boldsymbol{r}_{12}}\right] \times \\
& {\left[\int_{-\infty}^{0} \mathrm{~d} t^{\prime} \frac{\partial \Phi^{l}\left(\left|\boldsymbol{r}_{12}+\boldsymbol{g} t^{\prime}\right|\right)}{\partial \boldsymbol{r}_{12}}\right]\left\{\frac{\partial}{\partial \boldsymbol{v}_{1}}-\frac{\partial}{\partial \boldsymbol{v}_{2}}\right\} f_{1}\left(x_{1} ; t\right) f_{1}\left(x_{2} ; t\right) .}
\end{aligned}
$$

It is necessary to note that the quasiequilibrium binary correlation function $g_{2}$ takes into account total interaction potential (hard core part plus longrange Coulomb tail). 


\subsection{Local conservation laws in general form}

One of the major problem at correct derivation and solution to kinetic equations is its consistency with local conservation laws of particle density (or mass), momentum, total energy and substantiations of hydrodynamic equations and incomprehensible calculation of transport coefficients via molecular parameters. These conservation laws for classical systems in general have the following structure [69]:

$$
\begin{aligned}
& \frac{\partial}{\partial t} \rho(\boldsymbol{r} ; t)+\frac{\partial}{\partial \boldsymbol{r}} \boldsymbol{j}_{\rho}(\boldsymbol{r} ; t)=0, \\
& \frac{\partial}{\partial t} \boldsymbol{j}_{\rho}(\boldsymbol{r} ; t)+\frac{\partial}{\partial \boldsymbol{r}}: \stackrel{\leftrightarrow}{\Pi}(\boldsymbol{r} ; t)=0, \\
& \frac{\partial}{\partial t} \mathcal{E}(\boldsymbol{r} ; t)+\frac{\partial}{\partial \boldsymbol{r}} \boldsymbol{j}_{\mathcal{E}}(\boldsymbol{r} ; t)=0 .
\end{aligned}
$$

Here equation (2.5) is a mass conservation law (equation of continuity), equation (2.6) is a momentum conservation law, and equation (2.7) is that for total energy. Besides in these expressions $\rho(\boldsymbol{r} ; t)=m n(\boldsymbol{r} ; t)$ is a mass density, $n(\boldsymbol{r} ; t)$ is an average density of particle number

$$
\begin{aligned}
& n(\boldsymbol{r} ; t)=\langle\hat{n}(\boldsymbol{r} ; t)\rangle^{t}=\int \mathrm{d} \boldsymbol{v} f_{1}\left(x_{1} ; t\right), \\
& \hat{n}(\boldsymbol{r} ; t)=\sum_{a=1}^{N} \delta\left(\boldsymbol{r}-\boldsymbol{r}_{a}\right),
\end{aligned}
$$

where $\hat{n}(\boldsymbol{r} ; t)$ is a microscopic particle density; $\boldsymbol{j}_{\rho}(\boldsymbol{r} ; t)$ is an average momentum density, vector $\boldsymbol{V}(\boldsymbol{r} ; t)$ of average hydrodynamical velocity is related to $\boldsymbol{j}_{\rho}(\boldsymbol{r} ; t)$ by the expression:

$$
\begin{aligned}
& \boldsymbol{j}_{\rho}(\boldsymbol{r} ; t)=\rho(\boldsymbol{r} ; t) \boldsymbol{V}(\boldsymbol{r} ; t)=\left\langle\hat{\boldsymbol{j}}_{\rho}(\boldsymbol{r})\right\rangle^{t}=m \int \mathrm{d} \boldsymbol{v} \boldsymbol{v} f_{1}\left(x_{1} ; t\right), \\
& \hat{\boldsymbol{j}}_{\rho}(\boldsymbol{r})=m \sum_{a=1}^{N} \boldsymbol{v}_{a} \delta\left(\boldsymbol{r}-\boldsymbol{r}_{a}\right), \\
& \boldsymbol{V}(\boldsymbol{r} ; t)=\frac{1}{n(\boldsymbol{r} ; t)} \int \mathrm{d} \boldsymbol{v} \boldsymbol{v} f(x ; t),
\end{aligned}
$$

where $\hat{\boldsymbol{j}}_{\rho}(\boldsymbol{r})$ is a microscopic momentum density; $\Pi_{i j}(\boldsymbol{r} ; t)$ are components of a momentum density flow tensor:

$$
\begin{aligned}
& \Pi_{i j}(\boldsymbol{r} ; t)=\left\langle\hat{\Pi}_{i j}(\boldsymbol{r})\right\rangle^{t}=m \int \mathrm{d} \boldsymbol{v} v_{i} v_{j} f_{1}(x ; t)- \\
& \frac{1}{2} \int \mathrm{d} \boldsymbol{r}^{\prime} \mathrm{d} \boldsymbol{v} \mathrm{d} \boldsymbol{v}^{\prime}\left[\frac{\partial}{\partial r_{i}} \Phi\left(\left|\boldsymbol{r}-\boldsymbol{r}^{\prime}\right|\right)\right]\left(\boldsymbol{r}_{j}-\boldsymbol{r}_{j}^{\prime}\right) f_{2}\left(\boldsymbol{r}, \boldsymbol{r}^{\prime}, \boldsymbol{v}, \boldsymbol{v}^{\prime} ; t\right), \\
& \hat{\Pi}_{i j}(\boldsymbol{r})=\sum_{a=1}^{N}\left[m v_{a, i} v_{a, j}-\frac{1}{2} \sum_{b \neq a}^{N}\left[\frac{\partial}{\partial r_{a, i}} \Phi\left(\left|\boldsymbol{r}_{a b}\right|\right)\right] r_{a b, j}\right] \delta\left(\boldsymbol{r}-\boldsymbol{r}_{a}\right),
\end{aligned}
$$

where $\hat{\Pi}_{i j}(\boldsymbol{r})$ are components of a microscopic momentum density flow tensor; $\mathcal{E}(\boldsymbol{r} ; t)$ is an average density of total energy, which consists of kinetic 
and interaction parts:

$$
\begin{aligned}
& \mathcal{E}(\boldsymbol{r} ; t)=\mathcal{E}_{\text {kin }}(\boldsymbol{r} ; t)+\mathcal{E}_{\text {int }}(\boldsymbol{r} ; t), \\
& \mathcal{E}_{\text {kin }}(\boldsymbol{r} ; t)=\left\langle\hat{\mathcal{E}}_{k i n}(\boldsymbol{r})\right\rangle^{t}=\frac{m}{2} \int \mathrm{d} \boldsymbol{v} v^{2} f_{1}(x ; t), \\
& \mathcal{E}_{\text {int }}(\boldsymbol{r} ; t)=\left\langle\hat{\mathcal{E}}_{\text {int }}(\boldsymbol{r})\right\rangle^{t}=\frac{1}{2} \int \mathrm{d} \boldsymbol{r}^{\prime} \mathrm{d} \boldsymbol{v} \mathrm{d} \boldsymbol{v}^{\prime} \Phi\left(\left|\boldsymbol{r}-\boldsymbol{r}^{\prime}\right|\right) f_{2}\left(\boldsymbol{r}, \boldsymbol{r}^{\prime}, \boldsymbol{v}, \boldsymbol{v}^{\prime} ; t\right), \\
& \hat{\mathcal{E}}_{\text {kin }}(\boldsymbol{r})=\frac{m}{2} \sum_{a=1}^{N} v_{a}^{2} \delta\left(\boldsymbol{r}-\boldsymbol{r}_{a}\right), \\
& \hat{\mathcal{E}}_{\text {int }}(\boldsymbol{r})=\frac{1}{2} \sum_{a \neq b}^{N} \Phi\left(\left|\boldsymbol{r}_{a b}\right|\right) \delta\left(\boldsymbol{r}-\boldsymbol{r}_{a}\right),
\end{aligned}
$$

where $\hat{\mathcal{E}}_{\text {kin }}(\boldsymbol{r})$ is a microscopic density of kinetic energy, $\hat{\mathcal{E}}_{\text {int }}(\boldsymbol{r})$ is that of interaction energy; $\boldsymbol{j}_{\mathcal{E}}(\boldsymbol{r} ; t)$ is an average energy flow:

$$
\begin{aligned}
& \boldsymbol{j}_{\mathcal{E}}(\boldsymbol{r} ; t)=\left\langle\hat{\boldsymbol{j}}_{\mathcal{E}}(\boldsymbol{r})\right\rangle^{t}=\frac{m}{2} \int \mathrm{d} \boldsymbol{v} \boldsymbol{v} v^{2} f_{1}(x ; t)+ \\
& \quad \frac{1}{2} \int \mathrm{d} \boldsymbol{r}^{\prime} \mathrm{d} \boldsymbol{v} \mathrm{d} \boldsymbol{v}^{\prime} \boldsymbol{v} \Phi\left(\left|\boldsymbol{r}-\boldsymbol{r}^{\prime}\right|\right) f_{2}\left(\boldsymbol{r}, \boldsymbol{r}^{\prime}, \boldsymbol{v}, \boldsymbol{v}^{\prime} ; t\right)- \\
& \frac{1}{2} \int \mathrm{d} \boldsymbol{r}^{\prime} \mathrm{d} \boldsymbol{v} \mathrm{d} \boldsymbol{v}^{\prime}\left[\boldsymbol{v} \cdot \frac{\partial}{\partial \boldsymbol{r}} \Phi\left(\left|\boldsymbol{r}-\boldsymbol{r}^{\prime}\right|\right)\right]\left(\boldsymbol{r}-\boldsymbol{r}^{\prime}\right) f_{2}\left(\boldsymbol{r}, \boldsymbol{r}^{\prime}, \boldsymbol{v}, \boldsymbol{v}^{\prime} ; t\right), \\
& \hat{\boldsymbol{j}}_{\mathcal{E}}(\boldsymbol{r})=\sum_{a=1}^{N}\left(\frac{m v_{a}^{2}}{2}+\frac{1}{2} \sum_{a \neq b} \Phi\left(\left|\boldsymbol{r}_{a b}\right|\right)-\right. \\
& \left.\quad-\frac{1}{2} \sum_{a \neq b}^{N}\left[\frac{\partial}{\partial \boldsymbol{r}_{a}} \Phi\left(\left|\boldsymbol{r}_{a b}\right|\right)\right]\left(\boldsymbol{r}_{a}-\boldsymbol{r}_{b}\right)\right) \boldsymbol{v}_{a} \delta\left(\boldsymbol{r}-\boldsymbol{r}_{a}\right),
\end{aligned}
$$

where $\hat{\boldsymbol{j}}_{\mathcal{E}}(\boldsymbol{r})$ is its microscopic density.

It is important to underline, that the densities of particle number $n(\boldsymbol{r} ; t)$, momentum $\boldsymbol{j}_{\rho}(\boldsymbol{r} ; t)$ and kinetic energy $\mathcal{E}_{\text {kin }}(\boldsymbol{r} ; t)$ are defined via nonequilibrium one-particle distribution function $f_{1}(x ; t)$, whereas the density of interaction energy $\mathcal{E}_{\text {int }}(\boldsymbol{r} ; t)$ is represented through the nonequilibrium twoparticle distribution function $f_{2}\left(\boldsymbol{r}, \boldsymbol{r}^{\prime}, \boldsymbol{v}, \boldsymbol{v}^{\prime} ; t\right)$. Densities of momentum flow tensor $\overleftrightarrow{\Pi}(\boldsymbol{r} ; t)$ and energy flow vector $\boldsymbol{j}_{\mathcal{E}}(\boldsymbol{r} ; t)$ are expressed via one- and two-particle distribution functions.

One can exude corresponding convecting contributions into expressions for densities of momentum flow tensor $\stackrel{\leftrightarrow}{\Pi}(\boldsymbol{r} ; t)$ and energy flow vector $\boldsymbol{j}_{\mathcal{E}}(\boldsymbol{r} ; t)$ and passage from equations $(2.6),(2.7)$ to equations of hydrodynamics:

$$
\begin{aligned}
& \frac{\partial}{\partial t} j_{\rho}(\boldsymbol{r} ; t)+\frac{\partial}{\partial \boldsymbol{r}}[\rho(\boldsymbol{r} ; t) \boldsymbol{V} \boldsymbol{V}+\overleftrightarrow{P}(\boldsymbol{r} ; t)]=0, \\
& \frac{\partial}{\partial t}\left\{\rho(\boldsymbol{r} ; t)\left[\frac{V^{2}(\boldsymbol{r} ; t)}{2}+e(\boldsymbol{r} ; t)\right]\right\}+ \\
& \quad \frac{\partial}{\partial \boldsymbol{r}}\left[\boldsymbol{j}_{\rho}(\boldsymbol{r} ; t)\left[\frac{V^{2}(\boldsymbol{r} ; t)}{2}+e(\boldsymbol{r} ; t)\right]+\overleftrightarrow{P}(\boldsymbol{r} ; t) \boldsymbol{V}(\boldsymbol{r} ; t)+\boldsymbol{j}_{Q}(\boldsymbol{r} ; t)\right]=0,
\end{aligned}
$$


where $(2.8)$ is a macroscopic motion equation, in which $\overleftrightarrow{P}(\boldsymbol{r} ; t)$ is an average density of the pressure tensor

$$
\begin{aligned}
& P_{i j}(\boldsymbol{r} ; t)=\left\langle\hat{P}_{i j}(\boldsymbol{r})\right\rangle^{t}, \\
& \hat{P}_{i j}(\boldsymbol{r})=\sum_{a=1}^{N}\left[m\left(v_{a, i}-V_{i}(\boldsymbol{r} ; t)\right)\left(v_{a, j}-V_{j}(\boldsymbol{r} ; t)\right)-\right. \\
& \left.\frac{1}{2} \sum_{a \neq b}^{N}\left[\frac{\partial}{\partial r_{a, i}} \Phi\left(\left|\boldsymbol{r}_{a b}\right|\right)\right] r_{a b, j}\right] \delta\left(\boldsymbol{r}-\boldsymbol{r}_{a}\right) .
\end{aligned}
$$

Expression (2.9) is the equation for heat energy transfer, where $e(\boldsymbol{r} ; t)$ is an internal energy per mass unit, which is connected with total energy by relation

$$
\mathcal{E}(\boldsymbol{r} ; t)=\rho(\boldsymbol{r} ; t)\left[\frac{V^{2}(\boldsymbol{r} ; t)}{2}+e(\boldsymbol{r} ; t)\right] .
$$

$\boldsymbol{j}_{Q}(\boldsymbol{r} ; t)$ is a heat flow

$$
\begin{aligned}
& \boldsymbol{j}_{Q}(\boldsymbol{r} ; t)=\left\langle\hat{\boldsymbol{j}}_{Q}(\boldsymbol{r})\right\rangle^{t}, \\
& \hat{\boldsymbol{j}}_{Q}(\boldsymbol{r})=\frac{1}{2} \sum_{a=1}^{N}\left\{( \boldsymbol { v } _ { a } - \boldsymbol { V } ( \boldsymbol { r } ; t ) ) \left[m\left(\boldsymbol{v}_{a}-\boldsymbol{V}(\boldsymbol{r} ; t)\right)+\right.\right. \\
& \left.\left.\quad \sum_{a \neq b} \Phi\left(\left|\boldsymbol{r}_{a b}\right|\right)-\sum_{a \neq b}\left[\frac{\partial}{\partial \boldsymbol{r}_{a}} \Phi\left(\left|\boldsymbol{r}_{a b}\right|\right)\right]\left(\boldsymbol{r}_{a}-\boldsymbol{r}_{b}\right)\right]\right\} \delta\left(\boldsymbol{r}-\boldsymbol{r}_{a}\right) .
\end{aligned}
$$

In the case under consideration, for consistening of kinetics and hydrodynamics it is necessary to calculate average densities of momentum flow tensor $\stackrel{\leftrightarrow}{\Pi}(\boldsymbol{r} ; t)$ and energy flow vector $\boldsymbol{j}_{\mathcal{E}}(\boldsymbol{r} ; t)$ with the help of nonequilibrium distribution functions $f_{1}(x ; t), f_{2}\left(x, x^{\prime} ; t\right)$. Substitution of $f_{2}\left(x, x^{\prime} ; t\right)$ gives:

$$
\begin{aligned}
& \Pi_{i j}(\boldsymbol{r} ; t)=\int \mathrm{d} \boldsymbol{v} v_{i} v_{j} f_{1}\left(x_{1} ; t\right)- \\
& \frac{1}{2} \int \mathrm{d} \boldsymbol{r}^{\prime} \mathrm{d} \boldsymbol{v} \mathrm{d} \boldsymbol{v}^{\prime} \frac{\partial}{\partial r_{i}}\left(\Phi^{h s}\left(\left|\boldsymbol{r}-\boldsymbol{r}^{\prime}\right|\right)+\Phi^{l}\left(\left|\boldsymbol{r}-\boldsymbol{r}^{\prime}\right|\right)\right)\left(r_{j}-r_{j}^{\prime}\right) \times \\
& \varepsilon \int_{-\infty}^{t} \mathrm{~d} \tau \exp \left\{\left(\varepsilon+\mathrm{i} L_{2}\right) \tau\right\} g_{2}\left(\boldsymbol{r}, \boldsymbol{r}^{\prime} ; t+\tau\right) f_{1}(x ; t+\tau) f_{1}\left(x^{\prime} ; t+\tau\right), \\
& \boldsymbol{j}_{\mathcal{E}}(\boldsymbol{r} ; t)=\frac{1}{2} \int \mathrm{d} \boldsymbol{v} \boldsymbol{v} v^{2} f_{1}\left(x_{1} ; t\right)- \\
& \frac{1}{2} \int \mathrm{d} \boldsymbol{r}^{\prime} \mathrm{d} \boldsymbol{v} \mathrm{d} \boldsymbol{v}^{\prime} \boldsymbol{v}\left(\Phi^{h s}\left(\left|\boldsymbol{r}-\boldsymbol{r}^{\prime}\right|\right)+\Phi^{l}\left(\left|\boldsymbol{r}-\boldsymbol{r}^{\prime}\right|\right)\right) \times \\
& \varepsilon \int_{-\infty}^{t} \mathrm{~d} \tau \exp \left\{\left(\varepsilon+\mathrm{i} L_{2}\right) \tau\right\} g_{2}\left(\boldsymbol{r}, \boldsymbol{r}^{\prime} ; t+\tau\right) f_{1}(x ; t+\tau) f_{1}\left(x^{\prime} ; t+\tau\right)+ \\
& \frac{1}{2} \int \mathrm{d} \boldsymbol{r}^{\prime} \mathrm{d} \boldsymbol{v} \mathrm{d} \boldsymbol{v}^{\prime}\left(\boldsymbol{v} \cdot \frac{\partial}{\partial \boldsymbol{r}}\left(\Phi^{h s}\left(\left|\boldsymbol{r}-\boldsymbol{r}^{\prime}\right|\right)+\Phi^{l}\left(\left|\boldsymbol{r}-\boldsymbol{r}^{\prime}\right|\right)\right)\right)\left(\boldsymbol{r}-\boldsymbol{r}^{\prime}\right) \times
\end{aligned}
$$




$$
\varepsilon \int_{-\infty}^{t} \mathrm{~d} \tau \exp \left\{\left(\varepsilon+\mathrm{i} L_{2}\right) \tau\right\} g_{2}\left(\boldsymbol{r}, \boldsymbol{r}^{\prime} ; t+\tau\right) f_{1}(x ; t+\tau) f_{1}\left(x^{\prime} ; t+\tau\right) .
$$

For next calculations one should make here some approximations, namely the same, which have been used at derivation of Enskog-Landau kinetic equation [2,68]. First of all it is necessary to make an expansion over long-range part of the Liouville operator and to restrict oneself by linear components only:

$$
\mathrm{e}^{\mathrm{i} L_{2}^{l} \tau}=\mathrm{e}^{\mathrm{i} L_{2}^{0} \tau}+\int_{0}^{\tau} \mathrm{d} \lambda \mathrm{e}^{(\tau-\lambda) \mathrm{i} L_{2}^{0}}\left[\mathrm{i} L^{l}(1,2)\right] \mathrm{e}^{\lambda \mathrm{i} L_{2}^{0}}+\ldots
$$

Then taking into account that short-range interaction potential of hard spheres acts in region $[0 \div \sigma]$ and long-range interaction potential acts in region $[\sigma \div \infty]$, expressions for components of momentum density flow tensor $\overleftrightarrow{\Pi}(\boldsymbol{r} ; t)$ and density of total energy flow vector $\boldsymbol{j}_{\mathcal{E}}(\boldsymbol{r} ; t)$ read:

$$
\begin{aligned}
& \Pi_{i j}(\boldsymbol{r} ; t)=\int \mathrm{d} \boldsymbol{v} v_{i} v_{j} f_{1}\left(x_{1} ; t\right)- \\
& \frac{1}{2} \int_{0}^{\sigma} \mathrm{d} \boldsymbol{r}^{\prime} \mathrm{d} \boldsymbol{v} \mathrm{d} \boldsymbol{v}^{\prime} \frac{\partial}{\partial r_{i}}\left(\Phi^{h s}\left(\left|\boldsymbol{r}-\boldsymbol{r}^{\prime}\right|\right)\right)\left(r_{j}-r_{j}^{\prime}\right) \times \\
& \varepsilon \int_{-\infty}^{t} \mathrm{~d} \tau \exp \left\{\left(\varepsilon+\mathrm{i} L_{2}^{h s}\right) \tau\right\} g_{2}\left(\boldsymbol{r}, \boldsymbol{r}^{\prime} ; t+\tau\right) f_{1}(x ; t+\tau) f_{1}\left(x^{\prime} ; t+\tau\right)- \\
& \frac{1}{2} \int_{\sigma}^{\infty} \mathrm{d} \boldsymbol{r}^{\prime} \mathrm{d} \boldsymbol{v} \mathrm{d} \boldsymbol{v}^{\prime} \frac{\partial}{\partial r_{i}}\left(\Phi^{l}\left(\left|\boldsymbol{r}-\boldsymbol{r}^{\prime}\right|\right)\right)\left(r_{j}-r_{j}^{\prime}\right) \times \\
& \varepsilon \int_{-\infty}^{t} \mathrm{~d} \tau \exp \left\{\left(\varepsilon+\mathrm{i} L_{2}^{(0)}\right) \tau\right\} g_{2}\left(\boldsymbol{r}, \boldsymbol{r}^{\prime} ; t+\tau\right) f_{1}(x ; t+\tau) f_{1}\left(x^{\prime} ; t+\tau\right)- \\
& \frac{1}{2} \int_{\sigma}^{\infty} \mathrm{d} \boldsymbol{r}^{\prime} \mathrm{d} \boldsymbol{v} \mathrm{d} \boldsymbol{v}^{\prime} \frac{\partial}{\partial r_{i}}\left(\Phi^{l}\left(\left|\boldsymbol{r}-\boldsymbol{r}^{\prime}\right|\right)\right)\left(r_{j}-r_{j}^{\prime}\right) \times \\
& \varepsilon \int_{-\infty}^{t} \mathrm{~d} \tau \int_{0}^{\tau} \mathrm{d} \lambda \mathrm{e}^{(\tau-\lambda) \mathrm{i} L_{2}^{0}}\left[\mathrm{i} L^{l}(1,2)\right] \mathrm{e}^{\lambda \mathrm{i} L_{2}^{0} \times} \\
& g_{2}\left(\boldsymbol{r}, \boldsymbol{r}^{\prime} ; t+\tau\right) f_{1}(x ; t+\tau) f_{1}\left(x^{\prime} ; t+\tau\right), \\
& \boldsymbol{j}_{\mathcal{E}}(\boldsymbol{r} ; t)=\frac{1}{2} \int \mathrm{d} \boldsymbol{v} \boldsymbol{v} v^{2} f_{1}\left(x_{1} ; t\right)- \\
& \frac{1}{2} \int_{0}^{\sigma} \mathrm{d} \boldsymbol{r}^{\prime} \mathrm{d} \boldsymbol{v} \mathrm{d} \boldsymbol{v}^{\prime}\left(\boldsymbol{v} \cdot \frac{\partial}{\partial \boldsymbol{r}} \Phi^{h s}\left(\left|\boldsymbol{r}-\boldsymbol{r}^{\prime}\right|\right)\right)\left(\boldsymbol{r}-\boldsymbol{r}^{\prime}\right) \times
\end{aligned}
$$




$$
\begin{aligned}
& \varepsilon \int_{-\infty}^{t} \mathrm{~d} \tau \exp \left\{\left(\varepsilon+\mathrm{i} L_{2}^{h s}\right) \tau\right\} g_{2}\left(\boldsymbol{r}, \boldsymbol{r}^{\prime} ; t+\tau\right) f_{1}(x ; t+\tau) f_{1}\left(x^{\prime} ; t+\tau\right)+ \\
& \frac{1}{2} \int_{\sigma}^{\infty} \mathrm{d} \boldsymbol{r}^{\prime} \mathrm{d} \boldsymbol{v} \mathrm{d} \boldsymbol{v}^{\prime}\left(\boldsymbol{v} \cdot \frac{\partial}{\partial \boldsymbol{r}} \Phi^{l}\left(\left|\boldsymbol{r}-\boldsymbol{r}^{\prime}\right|\right)\right)\left(\boldsymbol{r}-\boldsymbol{r}^{\prime}\right) \times \\
& \varepsilon \int_{-\infty}^{t} \mathrm{~d} \tau \exp \left\{\left(\varepsilon+\mathrm{i} L_{2}^{(0)}\right) \tau\right\} g_{2}\left(\boldsymbol{r}, \boldsymbol{r}^{\prime} ; t+\tau\right) f_{1}(x ; t+\tau) f_{1}\left(x^{\prime} ; t+\tau\right)- \\
& \frac{1}{2} \int_{\sigma}^{\infty} \mathrm{d} \boldsymbol{r}^{\prime} \mathrm{d} \boldsymbol{v} \mathrm{d} \boldsymbol{v}^{\prime}\left(\boldsymbol{v} \cdot \frac{\partial}{\partial \boldsymbol{r}} \Phi^{l}\left(\left|\boldsymbol{r}-\boldsymbol{r}^{\prime}\right|\right)\right)\left(\boldsymbol{r}-\boldsymbol{r}^{\prime}\right) \times \\
& \varepsilon \int_{-\infty}^{t} \mathrm{~d} \tau \int_{0}^{\tau} \mathrm{d} \lambda \mathrm{e}^{(\tau-\lambda) \mathrm{i} L_{2}^{0}}\left[\mathrm{i} L^{l}(1,2)\right] \mathrm{e}^{\lambda \mathrm{i} L_{2}^{0} \times} \\
& g_{2}\left(\boldsymbol{r}, \boldsymbol{r}^{\prime} ; t+\tau\right) f_{1}(x ; t+\tau) f_{1}\left(x^{\prime} ; t+\tau\right)+ \\
& \frac{1}{2} \int_{0}^{\sigma} \mathrm{d} \boldsymbol{r}^{\prime} \mathrm{d} \boldsymbol{v} \mathrm{d} \boldsymbol{v}^{\prime} \boldsymbol{v} \Phi^{h s}\left(\left|\boldsymbol{r}-\boldsymbol{r}^{\prime}\right|\right) \times \\
& \varepsilon \int_{-\infty}^{t} \mathrm{~d} \tau \exp \left\{\left(\varepsilon+\mathrm{i} L_{2}^{h s}\right) \tau\right\} g_{2}\left(\boldsymbol{r}, \boldsymbol{r}^{\prime} ; t+\tau\right) f_{1}(x ; t+\tau) f_{1}\left(x^{\prime} ; t+\tau\right)+ \\
& \frac{1}{2} \int_{\sigma}^{\infty} \mathrm{d} \boldsymbol{r}^{\prime} \mathrm{d} \boldsymbol{v} \mathrm{d} \boldsymbol{v}^{\prime} \boldsymbol{v} \Phi^{l}\left(\left|\boldsymbol{r}-\boldsymbol{r}^{\prime}\right|\right) \times \\
& \varepsilon \int_{-\infty}^{t} \mathrm{~d} \tau \exp \left\{\left(\varepsilon+\mathrm{i} L_{2}^{(0)}\right) \tau\right\} g_{2}\left(\boldsymbol{r}, \boldsymbol{r}^{\prime} ; t+\tau\right) f_{1}(x ; t+\tau) f_{1}\left(x^{\prime} ; t+\tau\right)+ \\
& \frac{1}{2} \int_{\sigma}^{\infty} \mathrm{d} \boldsymbol{r}^{\prime} \mathrm{d} \boldsymbol{v} \mathrm{d} \boldsymbol{v}^{\prime} \boldsymbol{v} \Phi^{l}\left(\left|\boldsymbol{r}-\boldsymbol{r}^{\prime}\right|\right) \times \\
& \varepsilon \int_{-\infty}^{t} \mathrm{~d} \tau \int_{0}^{\tau} \mathrm{d} \lambda \mathrm{e}^{(\tau-\lambda) \mathrm{i} L_{2}^{0}}\left[\mathrm{i} L^{l}(1,2)\right] \mathrm{e}^{\lambda \mathrm{i} L_{2}^{0} \times} \\
& g_{2}\left(\boldsymbol{r}, \boldsymbol{r}^{\prime} ; t+\tau\right) f_{1}(x ; t+\tau) f_{1}\left(x^{\prime} ; t+\tau\right),
\end{aligned}
$$

where

$$
\begin{aligned}
& \mathrm{i} L_{2}^{h s, l}=\mathrm{i} L_{2}^{(0)}+\mathrm{i} L^{h s, l}(1,2), \\
& \mathrm{i} L_{2}^{(0)}=\boldsymbol{v} \frac{\partial}{\partial \boldsymbol{r}}+\boldsymbol{v}^{\prime} \frac{\partial}{\partial \boldsymbol{r}^{\prime}},
\end{aligned}
$$




$$
\mathrm{i} L^{h s, l}(1,2)=-\frac{1}{m} \frac{\partial}{\partial \boldsymbol{r}} \Phi^{h s, l}\left(\left|\boldsymbol{r}-\boldsymbol{r}^{\prime}\right|\right)\left\{\frac{\partial}{\partial \boldsymbol{v}}-\frac{\partial}{\partial \boldsymbol{v}^{\prime}}\right\},
$$

$f_{1}(x ; t)$ should be substituted into expressions as a solution to equation $(2.1)$.

\subsection{Equations for the parameters of reduced description}

To find the solution to the Enskog-Landau kinetic equation (2.1) using one or other method, there is necessary to take advantage of local conservation laws in corresponding approximations. In such a way the expressions for kinetic coefficients will be defined in a way of calculation of densities of momentum flow tensor $\overleftrightarrow{\Pi}(\boldsymbol{r} ; t)$ and energy flow vector $\boldsymbol{j}_{\mathcal{E}}(\boldsymbol{r} ; t)$ on the base of solution $f_{1}\left(x_{1} ; t\right)$ and corresponding approximations for $g_{2}\left(\boldsymbol{r}_{1}, \boldsymbol{r}_{2} ; t\right)$. So long as we find the solution that corresponds to linear hydrodynamical transport processes by gradients of thermodynamical parameters, the structure for expressions of densities of momentum flow tensor $\stackrel{\leftrightarrow}{\Pi}(\boldsymbol{r} ; t)$ and energy flow vector $\boldsymbol{j}_{\mathcal{E}}(\boldsymbol{r} ; t)$ should be defined immediately with the help of kinetic equation (2.1) without providing of calculations by already obtained formulas above. To this end it is convenient similarly to [2] to introduce the following hydrodynamical parameters: density $n\left(\boldsymbol{r}_{1} ; t\right)$ (or mass density $\rho\left(\boldsymbol{r}_{1} ; t\right)$ ), hydrodynamical velocity $\boldsymbol{V}\left(\boldsymbol{r}_{1} ; t\right)$ and density of kinetic energy $\omega_{k}\left(\boldsymbol{r}_{1} ; t\right)$ :

$$
\begin{aligned}
n\left(\boldsymbol{r}_{1} ; t\right) & =\int \mathrm{d} \boldsymbol{v}_{1} f_{1}\left(x_{1} ; t\right), \\
\boldsymbol{V}\left(\boldsymbol{r}_{1} ; t\right) & =\frac{1}{n\left(\boldsymbol{r}_{1} ; t\right)} \int \mathrm{d} \boldsymbol{v}_{1} f_{1}\left(x_{1} ; t\right) \boldsymbol{v}_{1}, \\
w_{k}\left(\boldsymbol{r}_{1} ; t\right) & =\frac{1}{2 n\left(\boldsymbol{r}_{1} ; t\right)} \int \mathrm{d} \boldsymbol{v}_{1} f_{1}\left(x_{1} ; t\right) c_{1}^{2}\left(\boldsymbol{r}_{1} ; t\right) .
\end{aligned}
$$

Here $\boldsymbol{c}_{1}\left(\boldsymbol{r}_{1} ; t\right)=\boldsymbol{v}_{1}-\boldsymbol{V}\left(\boldsymbol{r}_{1} ; t\right)$ is heat velocity of a particle. These parameters together constitute a 5 -component vector of interaction invariant $\Psi=\left(m, m \boldsymbol{v}, \omega_{k}\right)$. Multiplying initial kinetic equation (2.1) by components of vector $\Psi$ and integrating with respect to $\boldsymbol{v}_{1}$ one can obtain the equations for mentioned above hydrodynamical parameters:

$$
\begin{aligned}
\frac{1}{n\left(\boldsymbol{r}_{1} ; t\right)} \frac{\mathrm{d}}{\mathrm{d} t} n\left(\boldsymbol{r}_{1} ; t\right) & =-\frac{\partial}{\partial \boldsymbol{r}_{1}} \boldsymbol{V}\left(\boldsymbol{r}_{1} ; t\right), \\
\frac{1}{\rho\left(\boldsymbol{r}_{1} ; t\right)} \frac{\mathrm{d}}{\mathrm{d} t} \rho\left(\boldsymbol{r}_{1} ; t\right) & =-\frac{\partial}{\partial \boldsymbol{r}_{1}} \boldsymbol{V}\left(\boldsymbol{r}_{1} ; t\right), \\
\rho\left(\boldsymbol{r}_{1} ; t\right) \frac{\mathrm{d}}{\mathrm{d} t} \boldsymbol{V}\left(\boldsymbol{r}_{1} ; t\right) & =-\frac{\partial}{\partial \boldsymbol{r}_{1}}: \stackrel{\leftrightarrow}{P}\left(\boldsymbol{r}_{1} ; t\right), \\
\rho\left(\boldsymbol{r}_{1} ; t\right) \frac{\mathrm{d}}{\mathrm{d} t} w_{k}\left(\boldsymbol{r}_{1} ; t\right) & =-\frac{\partial}{\partial \boldsymbol{r}_{1}} \boldsymbol{q}\left(\boldsymbol{r}_{1} ; t\right)-\stackrel{\leftrightarrow}{P}\left(\boldsymbol{r}_{1} ; t\right): \frac{\partial}{\partial \boldsymbol{r}_{1}} \boldsymbol{V},
\end{aligned}
$$

where

$$
\begin{aligned}
& \stackrel{\leftrightarrow}{P}\left(\boldsymbol{r}_{1} ; t\right)=\stackrel{\leftrightarrow}{P}^{k}\left(\boldsymbol{r}_{1} ; t\right)+\stackrel{\leftrightarrow}{P}^{h s}\left(\boldsymbol{r}_{1} ; t\right)+\overleftrightarrow{\leftrightarrow}^{m f}\left(\boldsymbol{r}_{1} ; t\right)+\overleftrightarrow{P}^{l}\left(\boldsymbol{r}_{1} ; t\right) \\
& \boldsymbol{q}\left(\boldsymbol{r}_{1} ; t\right)=\boldsymbol{q}^{k}\left(\boldsymbol{r}_{1} ; t\right)+\boldsymbol{q}^{h s}\left(\boldsymbol{r}_{1} ; t\right)+\boldsymbol{q}^{m f}\left(\boldsymbol{r}_{1} ; t\right)+\boldsymbol{q}^{l}\left(\boldsymbol{r}_{1} ; t\right)
\end{aligned}
$$

are total stress tensor and vector of heat flow correspondingly. They have additive structure and contain several terms, each of them is stipulated by 
the influence from one of collision integrals: $\stackrel{\leftrightarrow}{P}^{h s}$ by Enskog collision integral (2.2), $\stackrel{\leftrightarrow}{P}^{m f}$ by collision integral of KMFT $(2.3), \stackrel{\leftrightarrow}{P}^{l}$ by Landau collision integral (2.4), $\stackrel{\leftrightarrow}{P}^{k}$ is pure kinetic contribution only. For the system under consideration one can present these quantities as follows:

$$
\begin{aligned}
& \stackrel{\leftrightarrow}{P}^{c}\left(\boldsymbol{r}_{1} ; t\right)=m \int \mathrm{d} \boldsymbol{v}_{1} f_{1}\left(x_{1} ; t\right) \boldsymbol{c}_{1}\left(\boldsymbol{r}_{1} ; t\right) \boldsymbol{c}_{1}\left(\boldsymbol{r}_{1} ; t\right) \\
& \stackrel{\leftrightarrow}{P}^{h s}\left(\boldsymbol{r}_{1} ; t\right)=\frac{m \sigma^{3}}{2} \int \mathrm{d} \boldsymbol{v}_{1} \mathrm{~d} \boldsymbol{v}_{2} \mathrm{~d} \hat{\boldsymbol{r}}_{12} \Theta\left(\hat{\boldsymbol{r}}_{12} \boldsymbol{g}\right)\left(\hat{\boldsymbol{r}}_{12} \boldsymbol{g}\right)\left\{\boldsymbol{v}_{1}^{\prime}-\boldsymbol{v}_{1}\right\} \hat{\boldsymbol{r}}_{12} \times \\
& \quad \int_{0}^{1} \mathrm{~d} \lambda F^{h s}\left(\boldsymbol{r}_{1}+\lambda \hat{\boldsymbol{r}}_{12} \sigma-\hat{\boldsymbol{r}}_{12} \sigma, \boldsymbol{v}_{1}, \boldsymbol{r}_{1}+\lambda \hat{\boldsymbol{r}}_{12} \sigma, \boldsymbol{v}_{2} ; t\right) \\
& \stackrel{\leftrightarrow}{P}^{m f}\left(\boldsymbol{r}_{1} ; t\right)=-\frac{1}{2} \int \mathrm{d} \boldsymbol{v}_{1} \mathrm{~d} \boldsymbol{v}_{2} \mathrm{~d} \boldsymbol{r}_{12}\left(\Phi^{l}\left(\left|\boldsymbol{r}_{12}\right|\right)\right)^{\prime} \frac{\boldsymbol{r}_{12} \boldsymbol{r}_{12}}{r_{12}} \times \\
& \int_{0}^{1} \mathrm{~d} \lambda F^{m f}\left(\boldsymbol{r}_{1}+\lambda \boldsymbol{r}_{12}-\boldsymbol{r}_{12}, \boldsymbol{v}_{1}, \boldsymbol{r}_{1}+\lambda \boldsymbol{r}_{12}, \boldsymbol{v}_{2} ; t\right)
\end{aligned}
$$

Here we use the notation

$$
\begin{aligned}
F^{h s} & \left(\boldsymbol{r}_{1}+\lambda \hat{\boldsymbol{r}}_{12} \sigma-\hat{\boldsymbol{r}}_{12} \sigma, \boldsymbol{v}_{1}, \boldsymbol{r}_{1}+\lambda \hat{\boldsymbol{r}}_{12} \sigma, \boldsymbol{v}_{2} ; t\right)= \\
& g_{2}\left(\boldsymbol{r}_{1}+\lambda \hat{\boldsymbol{r}}_{12} \sigma-\hat{\boldsymbol{r}}_{12} \sigma, \boldsymbol{r}_{1}+\lambda \hat{\boldsymbol{r}}_{12} \sigma ; t\right) \times \\
& f_{1}\left(\boldsymbol{r}_{1}+\lambda \hat{\boldsymbol{r}}_{12} \sigma-\hat{\boldsymbol{r}}_{12} \sigma, \boldsymbol{v}_{1} ; t\right) f_{1}\left(\boldsymbol{r}_{1}+\lambda \hat{\boldsymbol{r}}_{12} \sigma, \boldsymbol{v}_{2} ; t\right),
\end{aligned}
$$

$F^{m f}=F^{h s}$ at changing $\hat{\boldsymbol{r}}_{12} \sigma$ to $\boldsymbol{r}_{12}$. This difference in representation of $F^{h s}$ and $F^{m f}$ may be explained by the difference in scattering between hard spheres and Coulomb particles. Hard sphere scattering takes place at contact of particles only, while Coulomb particles can scatter at long distances.

To obtain $\overleftrightarrow{P}^{l}\left(\boldsymbol{r}_{1} ; t\right)$ one should transform first some terms under integrals in (2.4). First of all one should open an action of derivatives $\frac{\partial}{\partial \boldsymbol{r}_{12}}$; then calculate the integral with respect to $t^{\prime}$. Having long-range interaction potential $\Phi^{l}\left(\left|\boldsymbol{r}_{12}\right|\right)$ as a standard Coulomb one we can obtain:

$$
\frac{\partial}{\partial \boldsymbol{r}_{12}} \frac{1}{r_{12}}=-\frac{\boldsymbol{r}_{12}}{r_{12}^{3}}, \quad \frac{\partial}{\partial \boldsymbol{r}_{12}} \frac{1}{\left|\boldsymbol{r}_{12}+\boldsymbol{g} t^{\prime}\right|}=-\frac{\boldsymbol{r}_{12}}{r_{12}} \cdot \frac{r_{12}+g t^{\prime} \cos \left[\widehat{\boldsymbol{r}_{12} \boldsymbol{g}}\right]}{\left|\boldsymbol{r}_{12}+\boldsymbol{g} t^{\prime}\right|^{3}}
$$

where $\cos \left[\widehat{\boldsymbol{r}_{12} \boldsymbol{g}}\right]$ is a cosine of angle between the vector of mutual interparticle distance $\boldsymbol{r}_{12}$ and that of relative velocity $\boldsymbol{g}$. Integration of the last term with respect to $t^{\prime}$ gives:

$$
\int_{-\infty}^{0} \mathrm{~d} t^{\prime} \frac{r_{12}+g t^{\prime} \cos \left[\widehat{\boldsymbol{r}_{12} \boldsymbol{g}}\right]}{\left|\boldsymbol{r}_{12}+\boldsymbol{g} t^{\prime}\right|^{3}}=\frac{1}{g r_{12}} .
$$

Then the expression for Landau-like collision integral $I_{L}\left(x_{1} ; t\right)(2.4)$ takes form

$$
\begin{gathered}
I_{L}\left(x_{1} ; t\right)=\frac{Z^{4} e^{4}}{m^{2}} \frac{\partial}{\partial \boldsymbol{v}_{1}} \int \mathrm{d} x_{2} g_{2}\left(\boldsymbol{r}_{1}, \boldsymbol{r}_{1}+\boldsymbol{r}_{12} ; t\right) \frac{\boldsymbol{r}_{12} \boldsymbol{r}_{12}}{r_{12}^{5}} \frac{1}{g} \times \\
\left\{\frac{\partial}{\partial \boldsymbol{v}_{1}}-\frac{\partial}{\partial \boldsymbol{v}_{2}}\right\} f_{1}\left(x_{1} ; t\right) f_{1}\left(\boldsymbol{r}_{1}+\boldsymbol{r}_{12}, \boldsymbol{v}_{2} ; t\right) .
\end{gathered}
$$


One may pass from integration with respect to $\boldsymbol{r}_{2}$ to integration with respect into $\boldsymbol{r}_{12}$ by commutation $\boldsymbol{r}_{1}=\boldsymbol{r}_{1}, \boldsymbol{r}_{2}=\boldsymbol{r}_{12}-\boldsymbol{r}_{1}$. Finally for $\stackrel{\leftrightarrow}{P}^{l}\left(x_{1} ; t\right)$ one may obtain

$$
\begin{aligned}
& \stackrel{\leftrightarrow}{P}^{l}\left(\boldsymbol{r}_{1} ; t\right)= \\
& \quad \frac{Z^{4} e^{4}}{m} \int \mathrm{d} \boldsymbol{v}_{1} \boldsymbol{v}_{1} \frac{\partial}{\partial \boldsymbol{v}_{1}} \int \mathrm{d} x_{2} \frac{\boldsymbol{r}_{12} \boldsymbol{r}_{12}}{r_{12}^{5}} \cdot \frac{\boldsymbol{r}_{12}}{g}\left\{\frac{\partial}{\partial \boldsymbol{v}_{1}}-\frac{\partial}{\partial \boldsymbol{v}_{2}}\right\} \int_{0}^{1} \mathrm{~d} \lambda F^{l},
\end{aligned}
$$

where $F^{l}=F^{m f} . \stackrel{\leftrightarrow}{P}^{l}\left(\boldsymbol{r}_{1} ; t\right)$ is a new term in the structure of stress tensor (2.15) in comparison with results of [70]. Analysis of the structure for the expression (2.13) including (2.16) - (2.18), (2.20) and that for (2.6) together with the final form for $\stackrel{\leftrightarrow}{\Pi}\left(\boldsymbol{r}_{1} ; t\right)(2.10)$ allows to affirm that they contain identical terms. So, using of equations (2.12) and (2.13) at solving of kinetic equation (2.1) is equivalent to using equations (2.5) and (2.6).

Let us write heat flow vectors.

$$
\begin{gathered}
\boldsymbol{q}^{k}\left(\boldsymbol{r}_{1} ; t\right)=m \int \mathrm{d} \boldsymbol{v}_{1} f_{1}\left(x_{1} ; t\right) c_{1}^{2}\left(\boldsymbol{r}_{1} ; t\right) \boldsymbol{c}_{1}\left(\boldsymbol{r}_{1} ; t\right), \\
\boldsymbol{q}^{h s}\left(\boldsymbol{r}_{1} ; t\right)=\frac{m \sigma^{3}}{2} \int \mathrm{d} \boldsymbol{v}_{1} \mathrm{~d} \boldsymbol{v}_{2} \mathrm{~d} \hat{\boldsymbol{r}}_{12} \Theta\left(\hat{\boldsymbol{r}}_{12} \boldsymbol{g}\right)\left(\hat{\boldsymbol{r}}_{12} \boldsymbol{g}\right) \boldsymbol{r}_{12} \times \\
\left\{\left(c_{1}^{\prime}\left(\boldsymbol{r}_{1} ; t\right)\right)^{2}-\left(c_{1}\left(\boldsymbol{r}_{1} ; t\right)\right)^{2}\right\} \int_{0}^{1} \mathrm{~d} \lambda F^{h s} .
\end{gathered}
$$

Leaning on the symmetrical behaviour for a one-particle distribution function $f_{1}$, one can show even without available analytical form for $f_{1}\left(x_{1} ; t\right)$, that $\boldsymbol{q}^{h s}\left(\boldsymbol{r}_{1} ; t\right)=0$. Mean field term gives an influence on stress tensor only, the heat flow vector does not change its form. Relation for $\boldsymbol{q}^{l}\left(\boldsymbol{r}_{1} ; t\right)$ can be written by analogy with $\stackrel{\leftrightarrow}{P}^{l}\left(\boldsymbol{r}_{1} ; t\right)$ :

$$
\begin{aligned}
& \boldsymbol{q}^{l}\left(\boldsymbol{r}_{1} ; t\right)= \\
& \quad \frac{Z^{4} e^{4}}{2 m} \int \mathrm{d} \boldsymbol{v}_{1} c_{1}^{2} \frac{\partial}{\partial \boldsymbol{v}_{1}} \int \mathrm{d} x_{2} \frac{\boldsymbol{r}_{12} \boldsymbol{r}_{12}}{r_{12}^{5}} \cdot \frac{\boldsymbol{r}_{12}}{g}\left\{\frac{\partial}{\partial \boldsymbol{v}_{1}}-\frac{\partial}{\partial \boldsymbol{v}_{2}}\right\} \int_{0}^{1} \mathrm{~d} \lambda F^{l} .
\end{aligned}
$$

Expression (2.23) is also new in comparison with results of [70]. If to compare (2.14) and (2.7) supplemented by $(2.21)-(2.23),(2.20)$ and $(2.11)$ respectively we can find out some differences in their structure. In corresponding approximations the difference is due to two last terms in $\boldsymbol{j}_{\mathcal{E}}\left(\boldsymbol{r}_{1} ; t\right)$ (2.11). But they are not important because we consider approximation of linear hydrodynamics only. So, when obtaining an approximate solution to equation (2.1) it is sufficient in principle to more use of equations (2.12) (2.14).

\section{Boundary conditions method}

We shall construct a normal solution to the Enskog-Landau kinetic equation (2.1) using boundary conditions method [64,67]. Following this method let 
us bring into right hand side of equation (2.1) an infinity small source with $\varepsilon \rightarrow 0$ :

$$
\begin{aligned}
& \left\{\frac{\partial}{\partial t}+\boldsymbol{v}_{1} \frac{\partial}{\partial \boldsymbol{r}_{1}}\right\} f_{1}\left(x_{1} ; t\right)= \\
& I_{E}\left(x_{1} ; t\right)+I_{M F}\left(x_{1} ; t\right)+I_{L}\left(x_{1} ; t\right)-\varepsilon\left(f_{1}\left(x_{1} ; t\right)-f_{1}^{(0)}\left(x_{1} ; t\right)\right),
\end{aligned}
$$

where $f_{1}^{(0)}\left(x_{1} ; t\right)$ is some already known one-particle distribution function, satisfying equations $(2.12)-(2.14)$ for parameters of reduced description of our system. Then the solution can be found in the form $f_{1}\left(x_{1} ; t\right)=$ $f_{1}^{(0)}\left(x_{1} ; t\right)+\delta f\left(x_{1} ; t\right)$ and search of the normal solution implies treatment of the correction $\delta f\left(x_{1} ; t\right)$. Substituting $f_{1}\left(x_{1} ; t\right)$ into (3.1), one can obtain:

$$
\begin{gathered}
\left\{\frac{\partial}{\partial t}+\boldsymbol{v}_{1} \frac{\partial}{\partial \boldsymbol{r}_{1}}+\varepsilon\right\} \delta f+\frac{D}{D t} f_{1}^{(0)}=I_{M F}\left(f_{1}^{(0)}\right)+I_{M F}(\delta f)+ \\
I_{E}\left(f_{1}^{(0)}, f_{1}^{(0)}\right)+I_{L}\left(f_{1}^{(0)}, f_{1}^{(0)}\right)+I_{E}\left(f_{1}^{(0)}, \delta f\right)+I_{E}\left(\delta f, f_{1}^{(0)}\right)+ \\
I_{L}\left(f_{1}^{(0)}, \delta f\right)+I_{L}\left(\delta f, f_{1}^{(0)}\right)+I_{E}(\delta f, \delta f)+I_{L}(\delta f, \delta f) .
\end{gathered}
$$

Content and structure for used conventional signs are obvious [2,64,67]. Also the fact was taken into account about $I_{M F}\left(x_{1} ; t\right)$ collision integral $(2.3)$, which is a functional of one-particle distribution function only. Terms with the subscript $E$ are nonlocal, therefore in further calculations we should take their expansion with respect to the local one-particle distribution function and cut off this expansion by terms with degrees higher than $\delta f$. In the case when terms with subscripts $M F$ and $L$ also mean nonlocal functionals, one should apply mentioned above procedure to them too. Let us combine some terms in (3.2):

$$
\begin{array}{ll}
I_{E}(\delta f)=I_{E}\left(f_{1}^{(0)}, \delta f\right)+I_{E}\left(\delta f, f_{1}^{(0)}\right) & \begin{array}{l}
\text { linearized nonlocal Enskog col- } \\
\text { lision functional, }
\end{array} \\
I_{L}(\delta f)=I_{L}\left(f_{1}^{(0)}, \delta f\right)+I_{L}\left(\delta f, f_{1}^{(0)}\right) & \begin{array}{l}
\text { linearized Landau collision fun- } \\
\text { ctional. }
\end{array}
\end{array}
$$

Having these notations in mind one can transform equation (3.2) into the new form:

$$
\begin{aligned}
& \frac{\partial}{\partial t} \delta f-I_{E}^{(0)}(\delta f)-I_{M F}(\delta f)-I_{L}(\delta f)+\varepsilon \delta f=-\frac{D}{D t} f_{1}^{(0)}- \\
& \boldsymbol{v}_{1} \frac{\partial}{\partial \boldsymbol{r}_{1}} \delta f+I_{E}\left(f_{1}^{(0)}, f_{1}^{(0)}\right)+I_{M F}\left(f_{1}^{(0)}\right)+I_{L}\left(f_{1}^{(0)}, f_{1}^{(0)}\right)+I_{E}^{(1)}(\delta f) .
\end{aligned}
$$

Here $I_{E}^{(0)}(\delta f)$ is linearized local Enskog collision operator which is a zeroth term in expansion of linearized nonlocal collision functional $I_{E}(\delta f), I_{E}^{(1)}(\delta f)$ represents all other terms in this expansion (expansion goes on nonlocality).

Let us designate $L_{t}(\delta f)=I_{E}^{(0)}(\delta f)+I_{M F}(\delta f)+I_{L}(\delta f)$ and introduce an operator $S\left(t, t^{\prime}\right)$ with following properties:

$$
\frac{\partial}{\partial t} S\left(t, t^{\prime}\right)=L_{t}(\delta f) S\left(t, t^{\prime}\right),\left.\quad S\left(t, t^{\prime}\right)\right|_{t^{\prime}=t}=1 .
$$


Using these properties of operator $S\left(t, t^{\prime}\right)$ one can represent equation (3.3) in integral form. It reads:

$$
\begin{gathered}
\delta f\left(x_{1} ; t\right)=\int_{-\infty}^{t} \mathrm{~d} t^{\prime} \mathrm{e}^{-\varepsilon\left(t-t^{\prime}\right)} S\left(t, t^{\prime}\right)\left\{-\frac{D}{D t} f_{1}^{(0)}-\boldsymbol{v}_{1} \frac{\partial}{\partial \boldsymbol{r}_{1}} \delta f+\right. \\
\left.I_{E}\left(f_{1}^{(0)}, f_{1}^{(0)}\right)+I_{M F}\left(f_{1}^{(0)}\right)+I_{L}\left(f_{1}^{(0)}, f_{1}^{(0)}\right)+I_{E}^{(1)}(\delta f)\right\}_{t^{\prime}},
\end{gathered}
$$

where subscript $t^{\prime}$ at the bottom of right brase means that expression integrated is a function of $t^{\prime}$ (depends on $t^{\prime}$ ). An additional condition to find $\delta f(x ; t)$ is the evident limit $\lim _{t \rightarrow-\infty} \delta f(x ; t)=0$. Now it is easy to start itemizing procedure for determining the correction $\delta f(x ; t)$. For example, it could be organized in the following way:

$$
\begin{gathered}
\delta f^{(k+1)}\left(x_{1} ; t\right)=\int_{-\infty}^{t} \mathrm{~d} t^{\prime} \mathrm{e}^{-\varepsilon\left(t-t^{\prime}\right)} S\left(t, t^{\prime}\right)\left\{-\frac{D}{D t} f_{1}^{(0)}-\boldsymbol{v}_{1} \frac{\partial}{\partial \boldsymbol{r}_{1}} \delta f^{(k)}\right. \\
\left.+I_{E}\left(f_{1}^{(0)}, f_{1}^{(0)}\right)+I_{M F}\left(f_{1}^{(0)}\right)+I_{L}\left(f_{1}^{(0)}, f_{1}^{(0)}\right)+I_{E}^{(1)}\left(\delta f^{(k)}\right)\right\}_{t^{\prime}}
\end{gathered}
$$

by that $\left.\delta f\right|_{k=0}=0$ and to construct $(k+1)$-th approximation the laws are used (or equations for reduced description parameters) in $k$-th approximation. To realize this procedure it is necessary to define zeroth approximation for the one-particle distribution function $f_{1}^{(0)}\left(x_{1} ; t\right)$. In the case of spherical charged particles $f_{1}^{(0)}\left(x_{1} ; t\right)$ could be chosen as a local-equilibrium Maxwell distribution function

$$
f_{1}^{(0)}\left(x_{1} ; t\right)=n\left(\boldsymbol{r}_{1} ; t\right)\left(\frac{m}{2 \pi k T\left(\boldsymbol{r}_{1} ; t\right)}\right)^{3 / 2} \exp \left\{-\frac{m c_{1}^{2}\left(\boldsymbol{r}_{1} ; t\right)}{2 k T\left(\boldsymbol{r}_{1} ; t\right)}\right\} .
$$

Let us find correction to the distribution function $f_{1}^{(0)}\left(x_{1} ; t\right)$ using itemizing procedure (3.5). By calculating and obtaining of conservation laws $(2.12),(2.13)$ and equation (2.14), we should take into account the following relations:

$$
\begin{aligned}
& g_{2}\left(\boldsymbol{r}_{1}, \boldsymbol{r}_{2} ; t\right) \equiv g_{2}\left(\boldsymbol{r}_{1}, \boldsymbol{r}_{2} ; n(t), \beta(t)\right) \rightarrow g_{2}\left(\boldsymbol{r}_{12} ; n(t), \beta(t)\right), \\
& F \rightarrow F^{(0)}=g_{2}\left(\boldsymbol{r}_{12} ; n(t), \beta(t)\right) f_{1}^{(0)}\left(x_{1} ; t\right) f_{1}^{(0)}\left(\boldsymbol{r}_{1}, \boldsymbol{v}_{2} ; t\right),
\end{aligned}
$$

here $g_{2}\left(\boldsymbol{r}_{12} ; n(t), \beta(t)\right)$ is a binary quasiequilibrium correlation function, which depends on mutual distance between particles. It should be noted that together with the mentioned representation of a binary quasiequilibrium correlation function it can be introduced in another way, namely,

$$
\begin{aligned}
& g_{2}\left(\boldsymbol{r}_{1}, \boldsymbol{r}_{2} ; t\right) \equiv g_{2}\left(\boldsymbol{r}_{1}, \boldsymbol{r}_{2} ; n(t), \beta(t)\right)= \\
& \quad g_{2}\left(\boldsymbol{r}_{12}^{\prime} ; n(t), \beta(t)\right)+\frac{\boldsymbol{r}_{12}}{2} \frac{\partial}{\partial \boldsymbol{r}_{1}} g_{2}\left(\boldsymbol{r}_{12}^{\prime} ; n(t), \beta(t)\right)+\ldots
\end{aligned}
$$


and expansion of binary a quasiequilibrium correlation function is provided in the vicinity of the middle point $\boldsymbol{r}_{12}^{\prime}$

$$
\boldsymbol{r}_{12}^{\prime}=\boldsymbol{r}_{1}+\frac{\boldsymbol{r}_{12}}{2}, \text { whereas } \boldsymbol{r}_{12}=\boldsymbol{r}_{2}-\boldsymbol{r}_{1}
$$

We will use representation (3.6) below. Taking this into account let us write equations $(2.12)-(2.14)$ in the zeroth approximation. For the stress tensor and heat flow vector we obtain:

$$
\begin{array}{ll}
\overleftrightarrow{P}^{k}=\overleftrightarrow{I} P^{k}, & P^{k}=n k T, \\
\stackrel{\leftrightarrow}{P}^{h s}=\overleftrightarrow{I} P^{h s}, & P^{h s}=\frac{2}{3} \pi n^{2} \sigma^{3} k T g_{2}(\sigma \mid n, \beta), \\
\stackrel{\leftrightarrow}{P}^{m f}=\overleftrightarrow{I} P^{m f}, & P^{m f}=-\frac{2}{3} \pi(n Z e)^{2} \int_{\sigma}^{\infty} \frac{\mathrm{d} r}{r} g_{2}(r \mid n, \beta), \\
\overleftrightarrow{P}^{l}=0, & \boldsymbol{q}^{k}=\boldsymbol{q}^{h s}=\boldsymbol{q}^{m f}=\boldsymbol{q}^{l}=0 .
\end{array}
$$

In these expressions $\stackrel{\leftrightarrow}{I}$ is the unit tensor, $\stackrel{\leftrightarrow}{P}^{l}$ and $\boldsymbol{q}^{l}$ are equal to zero because the integration between symmetrical limits goes with odd function. Having calculated components $\overleftrightarrow{P}^{k}\left(\boldsymbol{r}_{1} ; t\right), \stackrel{\leftrightarrow}{P}^{h s}\left(\boldsymbol{r}_{1} ; t\right), \overleftrightarrow{P}^{m f}\left(\boldsymbol{r}_{1} ; t\right)$ and $\overleftrightarrow{P}^{l}\left(\boldsymbol{r}_{1} ; t\right)(3.7)-$ (3.10) one can write total pressure in the zeroth approximation:

$$
P=n k T\left(1+\frac{2}{3} \pi n \sigma^{3} g_{2}(\sigma \mid n, \beta)\right)-\frac{2}{3} \pi(n Z e)^{2} \int_{\sigma}^{\infty} \frac{\mathrm{d} r}{r} g_{2}(r \mid n, \beta) .
$$

Let us evaluate now expressions in brackets on the right-hand side in (3.5). It yields:

$$
\begin{aligned}
& \frac{D}{D t} f_{1}^{(0)}=f_{1}^{(0)}\left\{\frac{1}{n} \frac{\mathrm{d} n}{\mathrm{~d} t}+\left(\frac{m c_{1}^{2}}{2 k T}-\frac{3}{2}\right) \frac{1}{T} \frac{\mathrm{d} T}{\mathrm{~d} t}+\right. \\
& \left.\frac{m \boldsymbol{c}_{1}}{k T} \frac{\mathrm{d} \boldsymbol{V}}{\mathrm{d} t}+\left(\frac{m c_{1}^{2}}{2 k T}-\frac{3}{2}\right) \frac{\boldsymbol{c}_{1}}{T} \frac{\partial T}{\partial \boldsymbol{r}_{1}}+\frac{m}{k T} \boldsymbol{c}_{1} \boldsymbol{c}_{1}: \frac{\partial \boldsymbol{V}}{\partial \boldsymbol{r}_{1}}\right\} .
\end{aligned}
$$

Equations (2.12) - (2.14) in the zeroth approximation are used with taking account of $(3.7)-(3.10)$ for excluding of time derivatives in (3.11). To analyse next terms we should set $k=0$ :

$$
\begin{aligned}
& I_{E}^{(k+1)}\left(f_{1}^{(0)}, f_{1}^{(0)}\right)=-\frac{P^{h s}}{P^{k}} f_{1}^{(0)}\left\{2 \boldsymbol{c}_{1} \frac{\partial}{\partial \boldsymbol{r}_{1}} \ln n+\right. \\
& \left.\left(\frac{3 m c_{1}^{2}}{10 k T}-\frac{1}{2}\right) \boldsymbol{c}_{1} \frac{\partial}{\partial \boldsymbol{r}_{1}} \ln T+\frac{2 m}{5 k T} \boldsymbol{c}_{1} \boldsymbol{c}_{1}: \frac{\partial \boldsymbol{V}}{\partial \boldsymbol{r}_{1}}+\left(\frac{m c_{1}^{2}}{5 k T}-1\right) \frac{\partial \boldsymbol{V}}{\partial \boldsymbol{r}_{1}}\right\}, \\
& I_{M F}\left(f_{1}^{(0)}\right)=f_{1}^{(0)} \frac{\boldsymbol{c}_{1}}{P^{k}} \frac{\partial P^{m f}}{\partial \boldsymbol{r}_{1}}, I_{L}\left(f_{1}^{(0)}, f_{1}^{(0)}\right)=0, I_{E}^{(1)}\left(\delta f^{(k)}\right)=0 .
\end{aligned}
$$

So now one can write total expression for the correction $\delta f\left(x_{1} ; t\right)$ in the first approximation:

$$
\delta f^{(1)}\left(x_{1} ; t\right)=-\int_{-\infty}^{t} \mathrm{~d} t^{\prime} \mathrm{e}^{-\varepsilon\left(t-t^{\prime}\right)} S\left(t, t^{\prime}\right)\left[f_{1}^{(0)}\left(x_{1} ; t\right) \times\right.
$$




$$
\begin{aligned}
& \left\{\left(1+\frac{2}{5} \pi n \sigma^{3} g_{2}(\sigma \mid n, \beta)\right)\left[\frac{m c_{1}^{2}}{2 k T}-\frac{5}{2}\right] \boldsymbol{c}_{1} \frac{\partial}{\partial \boldsymbol{r}_{1}} \ln T\left(\boldsymbol{r}_{1} ; t\right)+\right. \\
& \left.\left.\left(1+\frac{4}{15} \pi n \sigma^{3} g_{2}(\sigma \mid n, \beta)\right) \frac{m}{k T}\left[\boldsymbol{c}_{1} \boldsymbol{c}_{1}-\frac{1}{3} c_{1}^{2} \stackrel{\leftrightarrow}{I}\right]: \frac{\partial}{\partial \boldsymbol{r}_{1}} \boldsymbol{V}\left(\boldsymbol{r}_{1} ; t\right)\right\}\right]_{t^{\prime}}
\end{aligned}
$$

Terms, related to short-range interactions only, contribute evidently into the correction in this approximation. Particle sizes take part here on contrary to the kinetic theory of dilute gases $[33,44,45]$, where particles are considered as a point-like objects. Nevertheless, influence from long-range part of interaction and so far from short-range part of interaction are present "hidden" in the operator $S\left(t, t^{\prime}\right)$ (as well through operator $L_{t}$ ). Formally, the expression (3.14) looks completely the same as the correction in [70]. But the difference is in the structure of the operator $S\left(t, t^{\prime}\right)$.

\section{Transport equations in the first approximation}

Having total expression for correction $\delta f\left(x_{1} ; t\right)$ in the first approximation (3.14) one can calculate conservation laws (2.12), (2.13) and equation (2.14) in the same approximation. To this end it is necessary first obtain evident relations for determining quantities $(2.16)-(2.18),(2.20)-(2.23)$ in which the correction (3.14) could be engaged. For $\stackrel{\leftrightarrow}{P}^{k 1}\left(\boldsymbol{r}_{1} ; t\right)$ we obtain:

$$
\stackrel{\leftrightarrow}{P}^{k 1}\left(r_{1} ; t\right)=\stackrel{\leftrightarrow}{I} P^{k}-\int_{-\infty}^{t} \mathrm{~d} t^{\prime} \mathrm{e}^{-\varepsilon\left(t-t^{\prime}\right)} M^{k}\left(t, t^{\prime}\right)[\overleftrightarrow{S}]_{t^{\prime}}
$$

where

$$
\left(S_{t}\right)_{\alpha \beta}=\frac{1}{2}\left(\frac{\partial V_{\alpha}\left(\boldsymbol{r}_{1} ; t\right)}{\partial r_{1, \beta}}+\frac{\partial V_{\beta}\left(\boldsymbol{r}_{1} ; t\right)}{\partial r_{1, \alpha}}\right)-\frac{1}{3}\left(\frac{\partial \boldsymbol{V}\left(\boldsymbol{r}_{1} ; t\right)}{\partial \boldsymbol{r}_{1}}: \stackrel{\leftrightarrow}{I}\right) I_{\alpha \beta}
$$

is a velocity shift tensor,

$$
\begin{aligned}
& M^{k}\left(t, t^{\prime}\right)=\frac{m}{5} \int \mathrm{d} \boldsymbol{v}_{1} \boldsymbol{c}_{1} \boldsymbol{c}_{1} S\left(t, t^{\prime}\right) \times \\
& \quad\left[f_{1}^{(0)}\left(x_{1} ; t\right)\left(1+\frac{4}{15} \pi n \sigma^{3} g_{2}(\sigma \mid n, \beta)\right) \frac{m}{k T}\left(\boldsymbol{c}_{1} \boldsymbol{c}_{1}-\frac{1}{3} c_{1}^{2} \stackrel{\leftrightarrow}{I}\right)\right]_{t^{\prime}}
\end{aligned}
$$

is a kernel of kinetic part of transport equations.

For calculating $\overleftrightarrow{P}^{h s}{ }^{1}\left(\boldsymbol{r}_{1} ; t\right), \overleftrightarrow{P}^{m f}{ }^{1}\left(\boldsymbol{r}_{1} ; t\right)$ and $\overleftrightarrow{P}^{l 1}\left(\boldsymbol{r}_{1} ; t\right)$ we have to expand $F^{h s}, F^{m f}$ and $F^{l}(2.19)$ on inhomogeneity and deviation $\delta f\left(x_{1} ; t\right)$ and keep in the series initial terms only. The expansion for $F^{h s}$ reads:

$$
\begin{aligned}
& \int_{0}^{1} \mathrm{~d} \lambda g_{2}\left(\boldsymbol{r}_{1}+\lambda \hat{\boldsymbol{r}}_{12} \sigma-\hat{\boldsymbol{r}}_{12} \sigma, \boldsymbol{r}_{1}+\lambda \hat{\boldsymbol{r}}_{12} \sigma ; t\right) \times \\
& f_{1}\left(\boldsymbol{r}_{1}+\lambda \hat{\boldsymbol{r}}_{12} \sigma-\hat{\boldsymbol{r}}_{12} \sigma, \boldsymbol{v}_{1} ; t\right) f_{1}\left(\boldsymbol{r}_{1}+\lambda \hat{\boldsymbol{r}}_{12} \sigma, \boldsymbol{v}_{2} ; t\right)= \\
& \frac{\sigma}{2} g_{2}(\sigma \mid n, \beta) f_{1}^{(0)}\left(x_{1} ; t\right) f_{1}^{(0)}\left(\boldsymbol{r}_{1}, \boldsymbol{v}_{2} ; t\right)\left(\hat{\boldsymbol{r}}_{12} \cdot \nabla\right) \ln \frac{f_{1}^{(0)}\left(x_{1} ; t\right)}{f_{1}^{(0)}\left(\boldsymbol{r}_{1}, \boldsymbol{v}_{2} ; t\right)}+ \\
& g_{2}(\sigma \mid n, \beta)\left\{f_{1}^{(0)}\left(x_{1} ; t\right) \delta f_{1}^{(0)}\left(\boldsymbol{r}_{1}, \boldsymbol{v}_{2} ; t\right)+\delta f_{1}^{(0)}\left(x_{1} ; t\right) f_{1}^{(0)}\left(\boldsymbol{r}_{1}, \boldsymbol{v}_{2} ; t\right)\right\} .
\end{aligned}
$$


To obtain $\stackrel{\leftrightarrow}{P}^{m f 1}\left(\boldsymbol{r}_{1} ; t\right), \overleftrightarrow{P}^{l 1}\left(\boldsymbol{r}_{1} ; t\right)$ one may use expansion (4.4) with changing replacement rule

$$
g_{2}(\sigma \mid n, \beta) \rightarrow g_{2}\left(\boldsymbol{r}_{12} \mid n, \beta\right), \quad \hat{\boldsymbol{r}}_{12} \rightarrow\left|\boldsymbol{r}_{12}\right|^{-1} \boldsymbol{r}_{12}, \quad \sigma \rightarrow\left|\boldsymbol{r}_{12}\right| .
$$

So we get:

$$
\begin{aligned}
& \stackrel{\leftrightarrow}{P}^{h s 1}\left(\boldsymbol{r}_{1} ; t\right)=\overleftrightarrow{P}^{h s}-\frac{4}{9} n^{2} \sigma^{4} g_{2}(\sigma \mid n, \beta) \sqrt{\pi m k T}\left[\frac{6}{5} \overleftrightarrow{S}+(\nabla \cdot \boldsymbol{V}) \stackrel{\leftrightarrow}{I}\right] \\
& -\frac{4}{15} \pi n \sigma^{3} g_{2}(\sigma \mid n, \beta) \int_{-\infty}^{t} \mathrm{~d} t^{\prime} \mathrm{e}^{-\varepsilon\left(t-t^{\prime}\right)} M^{k}\left(t, t^{\prime}\right)[\overleftrightarrow{S}]_{t^{\prime}}, \\
& \stackrel{\leftrightarrow}{P}^{m f 1}\left(\boldsymbol{r}_{1} ; t\right)=\overleftrightarrow{I} P^{m f} .
\end{aligned}
$$

Mean field influence into total stress tensor remains the same as in zeroth approximation. Similar situation arises as to $\stackrel{\leftrightarrow}{P}^{l 1}\left(\boldsymbol{r}_{1} ; t\right)$ :

$$
\stackrel{\leftrightarrow}{P}^{l 1}\left(\boldsymbol{r}_{1} ; t\right)=\overleftrightarrow{P}^{l}\left(\boldsymbol{r}_{1} ; t\right)=0
$$

Total expression for stress tensor in the first approximation is a sum of (4.1), (4.5), (4.6) and (4.7):

$$
\begin{gathered}
\stackrel{\leftrightarrow}{P}\left(\boldsymbol{r}_{1} ; t\right)=\stackrel{\leftrightarrow}{I} P\left(\boldsymbol{r}_{1} ; t\right)-\frac{4}{9} n^{2} \sigma^{4} g_{2}(\sigma \mid n, \beta) \sqrt{\pi m k T}\left[\frac{6}{5} \stackrel{\leftrightarrow}{S}+(\nabla \cdot \boldsymbol{V}) \stackrel{\leftrightarrow}{I}\right]- \\
\left(1+\frac{4}{15} \pi n \sigma^{3} g_{2}(\sigma \mid n, \beta)\right) \int_{-\infty}^{t} \mathrm{~d} t^{\prime} \mathrm{e}^{-\varepsilon\left(t-t^{\prime}\right)} M^{k}\left(t, t^{\prime}\right)[\overleftrightarrow{S}]_{t^{\prime}}
\end{gathered}
$$

The calculations for heat flow vectors give:

$$
\begin{aligned}
& \boldsymbol{q}^{k 1}\left(\boldsymbol{r}_{1} ; t\right)=-\int_{-\infty}^{t} \mathrm{~d} t^{\prime} \mathrm{e}^{-\varepsilon\left(t-t^{\prime}\right)} L^{k}\left(t, t^{\prime}\right)\left[\frac{1}{T} \nabla T\right]_{t^{\prime}}, \\
& \boldsymbol{q}^{h s 1}\left(\boldsymbol{r}_{1} ; t\right)=-\frac{2}{3} n^{2} \sigma^{4} g_{2}(\sigma \mid n, \beta) \sqrt{\frac{\pi k^{3} T}{m}} \nabla T\left(\boldsymbol{r}_{1} ; t\right)- \\
& \frac{2}{5} \pi n \sigma^{3} g_{2}(\sigma \mid n, \beta) \int_{-\infty}^{t} \mathrm{~d} t^{\prime} \mathrm{e}^{-\varepsilon\left(t-t^{\prime}\right)} L^{k}\left(t, t^{\prime}\right)\left[\frac{1}{T} \nabla T\right]_{t^{\prime}} \\
& \boldsymbol{q}^{l 1}\left(\boldsymbol{r}_{1} ; t\right)=\boldsymbol{q}^{l}\left(\boldsymbol{r}_{1} ; t\right)=0 .
\end{aligned}
$$

Here

$$
\begin{aligned}
& L^{k}\left(t, t^{\prime}\right)=\frac{1}{3} \int \mathrm{d} \boldsymbol{v}_{1} c_{1} \frac{m c_{1}^{2}}{2} S\left(t, t^{\prime}\right) \times \\
& \quad\left[f_{1}^{(0)}\left(x_{1} ; t\right)\left(1+\frac{2}{5} \pi n \sigma^{3} g_{2}(\sigma \mid n, \beta)\right)\left(\frac{m c_{1}^{2}}{2 k T}-\frac{5}{2}\right) c_{1}\right]_{t^{\prime}}
\end{aligned}
$$


is other kernel of kinetic part of transport equations. Total expression for heat flux vector is a sum of $(4.8)-(4.10)$ :

$$
\begin{aligned}
& \boldsymbol{q}\left(\boldsymbol{r}_{1} ; t\right)=-\frac{2}{3} n^{2} \sigma^{4} g_{2}(\sigma \mid n, \beta) \sqrt{\frac{\pi k^{3} T}{m}} \nabla T\left(\boldsymbol{r}_{1} ; t\right)- \\
&\left(1+\frac{2}{5} \pi n \sigma^{3} g_{2}(\sigma \mid n, \beta)\right) \int_{-\infty}^{t} \mathrm{~d} t^{\prime} \mathrm{e}^{-\varepsilon\left(t-t^{\prime}\right)} L^{k}\left(t, t^{\prime}\right)\left[\frac{1}{T} \nabla T\right]_{t^{\prime}} .
\end{aligned}
$$

\section{Stationary process. Transport coefficients}

Let us consider a stationary process, when the operator $L_{t}$ does not depend on time, i.e. $S\left(t, t^{\prime}\right)=\exp \left\{L_{t}\left(t-t^{\prime}\right)\right\}$. Some terms in expressions for $\overleftrightarrow{P}\left(\boldsymbol{r}_{1} ; t\right)$ and $\boldsymbol{q}\left(\boldsymbol{r}_{1} ; t\right)$ can acquire simpler form. Third term in (4.5) and second term in (4.9) both right hand side appear as relations

$$
\begin{aligned}
& -\lim _{\varepsilon \rightarrow 0} \frac{1}{5} \int \mathrm{d} \boldsymbol{c}_{1} m \boldsymbol{c}_{1} \boldsymbol{c}_{1} \stackrel{\leftrightarrow}{B}\left(\boldsymbol{c}_{1}\right): \overleftrightarrow{S}, \\
& -\lim _{\varepsilon \rightarrow 0} \frac{1}{3} \int \mathrm{d} \boldsymbol{c}_{1} c_{1 \alpha} \frac{m c_{1}^{2}}{2} A_{\alpha}\left(\boldsymbol{c}_{1}\right) \nabla T,
\end{aligned}
$$

where $\overleftrightarrow{B}\left(\boldsymbol{c}_{1}\right)$ and $\boldsymbol{A}\left(\boldsymbol{c}_{1}\right)$ are defined in following way:

$$
\begin{gathered}
\overleftrightarrow{B}\left(\boldsymbol{c}_{1}\right)=\left(1+\frac{4}{15} \pi n \sigma^{3} g_{2}(\sigma \mid n, \beta)\right) \times \\
\int_{-\infty}^{0} \mathrm{~d} \tau \mathrm{e}^{-\tau \varepsilon} \mathrm{e}^{-\tau L_{t}} f_{1}^{(0)}\left(x_{1} ; t\right) \frac{m}{k T}\left(\boldsymbol{c}_{1} \boldsymbol{c}_{1}-\frac{1}{3} \boldsymbol{c}_{1}^{2} \stackrel{\leftrightarrow}{I}\right), \\
\boldsymbol{A}\left(\boldsymbol{c}_{1}\right)=\left(1+\frac{2}{5} \pi n \sigma^{3} g_{2}(\sigma \mid n, \beta)\right) \times \\
\int_{-\infty}^{0} \mathrm{~d} \tau \mathrm{e}^{-\tau \varepsilon} \mathrm{e}^{-\tau L_{t}} f_{1}^{(0)}\left(x_{1} ; t\right) \frac{\boldsymbol{c}_{1}}{T}\left(\frac{m c_{1}^{2}}{2 k T}-\frac{5}{2}\right) .
\end{gathered}
$$

Let us consider functions

$$
\begin{aligned}
& \overleftrightarrow{b}(\tau)=\mathrm{e}^{-\tau L_{t}} f_{1}^{(0)}\left(x_{1} ; t\right)\left[1+\frac{4}{15} \pi n \sigma^{3} g_{2}(\sigma \mid n, \beta)\right] \frac{m}{k T}\left[\boldsymbol{c}_{1} \boldsymbol{c}_{1}-\frac{1}{3} c_{1}^{2} I\right], \\
& \boldsymbol{a}(\tau)=\mathrm{e}^{-\tau L_{t}} f_{1}^{(0)}\left(x_{1} ; t\right)\left[1+\frac{2}{5} \pi n \sigma^{3} g_{2}(\sigma \mid n, \beta)\right] \frac{\boldsymbol{c}_{1}}{T}\left[\frac{m c_{1}^{2}}{2 k T}-\frac{5}{2}\right] .
\end{aligned}
$$

Differentiating expressions (5.2) for $\overleftrightarrow{b}(\tau)$ and $\boldsymbol{a}(\tau)$ with respect to $\tau$ one can obtain a set of equations:

$$
\frac{\partial \overleftrightarrow{b}}{\partial \tau}+L_{t} \stackrel{\leftrightarrow}{b}=0, \quad \frac{\partial \boldsymbol{a}}{\partial \tau}+L_{t} \boldsymbol{a}=0
$$


with initial conditions

$$
\begin{aligned}
& \overleftrightarrow{b}(0)=f_{1}^{(0)}\left(x_{1} ; t\right)\left(1+\frac{4}{15} \pi n \sigma^{3} g_{2}(\sigma \mid n, \beta)\right) \frac{m}{k T}\left(\boldsymbol{c}_{1} \boldsymbol{c}_{1}-\frac{1}{3} c_{1}^{2} \stackrel{\leftrightarrow}{I}\right), \\
& \boldsymbol{a}(0)=f_{1}^{(0)}\left(x_{1} ; t\right)\left(1+\frac{2}{5} \pi n \sigma^{3} g_{2}(\sigma \mid n, \beta)\right) \frac{\boldsymbol{c}_{1}}{T}\left(\frac{m c_{1}^{2}}{2 k T}-\frac{5}{2}\right) .
\end{aligned}
$$

Initial values $\overleftrightarrow{b}(0), \boldsymbol{a}(0)$ are known as expressions, which appears at solving kinetic equations in the theory of hard spheres RET [13,14]. It is also known, that these expressions are strictly orthogonal to 5-component vector $\Psi$ of additive invariants. Following [64] one can write

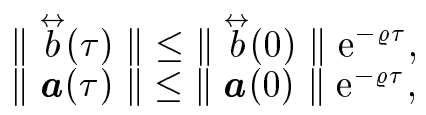

where $\|\varphi\|=\int \mathrm{d} \boldsymbol{c}_{1}\left[f_{1}^{(0)}\left(x_{1} ; t\right)\right]^{-1} \varphi^{2}$, and $\varrho<0$ is the nearest to zero self value, or point in a spectrum of the operator $L_{t}$. Due to this condition

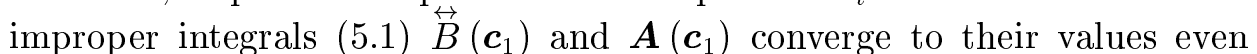
at $\varepsilon=0$, therefore the limiting transition $\varepsilon \rightarrow 0$ and integration in these relations are interchangeable and the limiting transition can be performed under integration. In response to this we get:

$$
\begin{array}{ll}
\frac{1}{5} \int \mathrm{d} \boldsymbol{c}_{1} m \boldsymbol{c}_{1} \boldsymbol{c}_{1} \stackrel{\leftrightarrow}{B}\left(\boldsymbol{c}_{1}\right): \overleftrightarrow{S}, \quad \text { where } \quad \stackrel{\leftrightarrow}{B}\left(\boldsymbol{c}_{1}\right)=\int_{-\infty}^{0} \mathrm{~d} \tau \stackrel{\leftrightarrow}{b}(\tau), \\
\frac{1}{3} \int \mathrm{d} \boldsymbol{c}_{1} c_{1, \alpha} \frac{m c_{1}^{2}}{2} A_{\alpha}\left(\boldsymbol{c}_{1}\right) \nabla T, \quad \text { where } \quad \boldsymbol{A}\left(\boldsymbol{c}_{1}\right)=\int_{-\infty}^{0} \mathrm{~d} \tau \boldsymbol{a}(\tau) .
\end{array}
$$

Integrating differential equations (5.3) between limits $-\infty$ to 0 with taking into consideration limiting conditions and as well as new relations (5.4) for $\overleftrightarrow{B}\left(\boldsymbol{c}_{1}\right)$ and $\boldsymbol{A}\left(\boldsymbol{c}_{1}\right)$, one can obtain integral equations for finding $\overleftrightarrow{B}\left(\boldsymbol{c}_{1}\right)$ and $\boldsymbol{A}\left(\boldsymbol{c}_{1}\right)$ :

$$
\begin{aligned}
& L_{t} \stackrel{\leftrightarrow}{B}=-\left[1+\frac{4}{15} \pi n \sigma^{3} g_{2}(\sigma \mid n, \beta)\right] \frac{m}{k T}\left[\boldsymbol{c}_{1} \boldsymbol{c}_{1}-\frac{1}{3} c_{1}^{2} \stackrel{\leftrightarrow}{I}\right] f_{1}^{(0)}\left(x_{1} ; t\right), \\
& L_{t} \boldsymbol{A}=-\left[1+\frac{2}{5} \pi n \sigma^{3} g_{2}(\sigma \mid n, \beta)\right] \frac{\boldsymbol{c}_{1}}{T}\left[\frac{m c_{1}^{2}}{2 k T}-\frac{5}{2}\right] f_{1}^{(0)}\left(x_{1} ; t\right) .
\end{aligned}
$$

Furthermore, similar reasonings also may be used for obtaining an expression for correction $\delta f\left(x_{1} ; t\right)$ in the stationary case. We have:

$$
\delta f_{1}\left(x_{1} ; t\right)=-\overleftrightarrow{B}: \frac{\partial \boldsymbol{V}\left(\boldsymbol{r}_{1} ; t\right)}{\partial \boldsymbol{r}_{1}}-\boldsymbol{A} \cdot \nabla T\left(\boldsymbol{r}_{1} ; t\right)
$$

The normal solution to the Enskog-Landau kinetic equation for onecomponent system of charged hard spheres with the help of ChapmanEnskog method in the case, when in long-range part of collision integral we put $g_{2}(\sigma \mid n, \beta) \rightarrow 1$, gives the following results [60] for stress tensor $\overleftrightarrow{P}\left(\boldsymbol{r}_{1} ; t\right)$ and heat flow vector $\boldsymbol{q}\left(\boldsymbol{r}_{1} ; t\right)$ :

$$
\overleftrightarrow{P}\left(\boldsymbol{r}_{1} ; t\right)=P\left(\boldsymbol{r}_{1} ; t\right) \stackrel{\leftrightarrow}{I}-2 \eta \overleftrightarrow{S}\left(\boldsymbol{r}_{1} ; t\right)-æ \nabla: \boldsymbol{V}\left(\boldsymbol{r}_{1} ; t\right)
$$




$$
\boldsymbol{q}\left(\boldsymbol{r}_{1} ; t\right)=-\lambda \nabla T\left(\boldsymbol{r}_{1} ; t\right),
$$

where $æ$ is a bulk viscosity

$$
æ=\frac{4}{9} \sqrt{\pi m k T} n^{2} \sigma^{4} g_{2}(\sigma \mid n, \beta),
$$

$\eta$ is a shear viscosity

$$
\eta=\frac{3}{5} æ+\frac{5}{8} \sqrt{\frac{m k T}{\pi}} \frac{\left\{1+\frac{4}{15} \pi n \sigma^{3} g_{2}(\sigma \mid n, \beta)\right\}^{2}}{2 \sigma^{2} g_{2}(\sigma \mid n, \beta)+\pi^{2} \frac{(Z e)^{4}}{(k T)^{2}} \ln \frac{D}{\sigma}},
$$

$\lambda$ is a thermal conductivity

$$
\lambda=\frac{3 k}{2 m} æ+\frac{75 k}{32 m} \sqrt{\frac{m k T}{\pi}} \frac{\left\{1+\frac{2}{5} \pi n \sigma^{3} g_{2}(\sigma \mid n, \beta)\right\}^{2}}{2 \sigma^{2} g_{2}(\sigma \mid n, \beta)+\pi^{2} \frac{(Z e)^{4}}{(k T)^{2}} \ln \frac{D}{\sigma}},
$$

$D$ is a screening radius in the system (an analogue of Debye radius with taking into account of particle size [60]).

One may remark that bulk viscosity has the same structure as in Chapman-Enskog method (5.5). But other transport coefficients have some distinctions from (5.6), (5.7). They are:

$$
\begin{aligned}
& \eta=\frac{3}{5} æ+2 n k T \frac{\left\{1+\frac{4}{15} \pi n \sigma^{3} g_{2}(\sigma \mid n, \beta)\right\}^{2}}{\left\{I_{E}^{(0)}(\delta f)+I_{L}(\delta f)\right\}}, \\
& \lambda=\frac{3 k}{2 m} æ+\frac{5 k}{m} n k T \frac{\left\{1+\frac{2}{5} \pi n \sigma^{3} g_{2}(\sigma \mid n, \beta)\right\}^{2}}{\left\{I_{E}^{(0)}(\delta f)+I_{L}(\delta f)\right\}} .
\end{aligned}
$$

Then the problem lies in calculating collision integrals $I_{E}^{(0)}(\delta f)$ and $I_{L}(\delta f)$, this means we should calculate collision integrals (2.2) (in the zeroth approximation on inhomogeneity) and (2.4) together in the first approximation on deviation $\delta f$, where $\delta f$ is substituted from (3.14). The matter of some difficulty is that correction (3.14) in its turn is expressed also via collision integrals $I_{E}^{(0)}(\delta f), I_{E}(\delta f)$, which are in operator $S\left(t, t^{\prime}\right)$. Let us remember, that operator $S\left(t, t^{\prime}\right)$ in the stationary case reads $S\left(t, t^{\prime}\right)=\exp \left\{L_{t}\left(t-t^{\prime}\right)\right\}$, where $L_{t}=I_{E}^{(0)}(\delta f)+I_{M F}(\delta f)+I_{L}(\delta f)$. So the first acceptable approximation should be that, when the correction $\delta f(3.14)$ is expressed via $I_{E}^{(0)}(\delta f)$, $I_{L}(\delta f)$ calculated with $\delta f^{\prime}$, where $\delta f^{\prime}=\delta f$ at $S\left(t, t^{\prime}\right)=1$. For $I_{E}^{(0)}(\delta f)$ we obtain the results [2], for $I_{L}(\delta f)$ in (5.8), (5.9) we can obtain the following:

$$
\begin{aligned}
& I_{L}(\delta f)=\frac{Z^{4} e^{4}}{m^{2}} \frac{\partial}{\partial \boldsymbol{v}_{1}} \int \mathrm{d} \boldsymbol{r}_{12} \mathrm{~d} \boldsymbol{v}_{2} g_{2}\left(\boldsymbol{r}_{1}, \boldsymbol{r}_{1}+\boldsymbol{r}_{12} ; t\right) \frac{\boldsymbol{r}_{12} \boldsymbol{r}_{12}}{r_{12}^{5}} \frac{1}{g}\left\{\frac{\partial}{\partial \boldsymbol{v}_{1}}-\frac{\partial}{\partial \boldsymbol{v}_{2}}\right\} \\
& \quad \times\left\{f_{1}\left(x_{1} ; t\right) \delta f\left(\boldsymbol{r}_{1}+\boldsymbol{r}_{12}, \boldsymbol{v}_{2} ; t\right)+\delta f\left(x_{1} ; t\right) f_{1}\left(\boldsymbol{r}_{1}+\boldsymbol{r}_{12}, \boldsymbol{v}_{2} ; t\right)\right\},
\end{aligned}
$$


where $\delta f(x ; t)$ is calculated via (3.14) with

$$
S\left(t, t^{\prime}\right)=\exp \left\{L_{t}\left(t-t^{\prime}\right)\right\}=\exp \left\{\left[I_{E}^{(0)}\left(\delta f^{\prime}\right)+I_{L}\left(\delta f^{\prime}\right)\right]\left(t-t^{\prime}\right)\right\}
$$

at $\delta f^{\prime}(x ; t)=\left.\delta f(x ; t)\right|_{S\left(t, t^{\prime}\right)=1}$. This stage of calculations needs explicit form for the binary quasiequilibrium correlation function $g_{2}$ both on the contact and in $\boldsymbol{r}$-space.

The reason for these differences were discussed in details [64] and could be transferred on results of this paper. At constructing of the normal solution for the Enskog-Landau kinetic equation time derivatives $\frac{\partial}{\partial t}$ of hydrodynamical parameters of reduced description don't vanish. Therefore the normal solution for this equation could be used for hydrodynamic description of fast processes.

\section{References}

[1] Zubarev D.N., Morozov V.G. Formulation of boundary conditions for the Bogolubov hierarchy with allowance for local conservation laws. / / Teor. Mat. Fiz., 1984, vol. 60, p. 270 (in Russian).

[2] Zubarev D.N., Morozov V.G., Omelyan I.P., Tokarchuk M.V. On kinetic equations for dense gases and liquids. // Teor. Mat. Fiz., 1991, vol. 87, No 1, p. 113-129 (in Russian).

[3] Zubarev D.N., Morozov V.G., Omelyan I.P., Tokarchuk M.V. The unification of kinetic and hydrodynamic approaches in the theory of dense gases and liquids. // Teor. Mat. Fiz., 1993, vol. 96, No 3, p. 325-350 (in Russian).

[4] Klimontovich Yu.L. On the necessity and possibility of the united description of kinetic and hydrodynamic processes. // Teor. Mat. Fiz. 1992, vol. 92, No 2, p. 312-330 (in Russian).

[5] Klimontovich Yu.L. The united description on kinetic and hydrodynamic processes in gases and plasmas. // Phys. Lett. A, 1992, vol. 170, p. 434.

[6] Bogolubov N.N. Problems of a dynamical theory in statistical physics, in: Studies in statistical mechanics, vol. 1 (eds. J. de Boer and G.E.Uhlenbeck). Amsterdam, North-Holland, 1962.

[7] Born M., Green H.S. A general kinetic theory of liquids. Cambridge, Cambridge University Press, 1949.

[8] Kirkwood J.G. // Journ. Chem. Phys., 1946, vol. 14, p. 180; 1947, vol. 15, p. 72.

[9] Yvon J. La théorie des fluids et l'équation d'etat: actualités scientificues et industrielles. Paris, Hermann and Cie, 1935.

[10] Zubarev D.N., Novikov M.Yu. Generalized formulation of the boundary condition for the Liouville equation and for BBGKY hierarchy. // Teor. Mat. Fiz. 1972, vol. 13, No 3, p. 406-420 (in Russian).

[11] Chapman S., Cowling T.G., The mathematical theory of nonuniform gases. Cambridge, Cambridge University Press, 1952.

[12] Cercignani C. Mathematical methods in kinetic theory. New York, Plenum Press, 1969.

[13] Ferziger J.H., Kaper H.G. Mathematical theory of transport processes in gases. Amsterdam, North-Holland, 1972.

[14] Ernst M.H., van Beijeren H. The modified Enskog equation. // Physica, 1973, vol. 68, No 3, p. 437-456.

[15] Karkheck J., Stell G. Kinetic mean-field theories. // J. Chem. Phys., 1981, vol. 75, p. 1475-1487.

[16] Stell G., Karkheck J., van Beijeren H. Kinetic mean-field theories: Results of energy constraint in maximizing entropy. // J. Chem. Phys., 1983, vol 79, No 6, p. 3166-3176. 
[17] Davis H.T., Rice S.A., Sangers J.V. On the kinetic theory of dense fluids. The fluid of rigid spheres with a squarewell attraction. // J. Chem. Phys., 1961, vol 35, No 6, p. 2210-2233.

[18] Karkheck J., van Beijeren H., de Schepper J., Stell G. Kinetic theory and $H$-theorem for a dense square-well fluid. // Phys. Rev. A, 1985, vol.32, No 4, p. 2517-2520.

[19] Rudyak V.Ya. On the theory of kinetic equations for dense gas. // Jurn. Tehn. Fiz. $^{1}$, 1984, vol. 54, No 7, p. 1246-1252 (in Russian).

[20] Rudyak V.Ya. About derivation of Enskog-like kinetic equation for dense gas. // Teplofiz. Vys. Temp. ${ }^{2}, 1985$, vol. 23, No 2, p. 268-272 (in Russian).

[21] Rudyak V.Ya., Yanenko N.N. On taking into account intermolecular attractive forces at the derivation of kinetic equations. // Teor. Mat. Fiz., 1985, vol. 64, No 2, p. 277-286 (in Russian).

[22] Rudyak V.Ya. Kinetic equations of nonideal gas with real interaction potentials. // Jurn. Tehn. Fiz., 1987, vol. 57, No 8, p. 1466-1475 (in Russian).

[23] Rudyak V.Ya. Transport coefficients for nonideal gas. // Teplofiz. Vys. Temp., 1989, vol. 27, No 4, p. 697-701 (in Russian).

[24] Rudyak V.Ya. Statistical theory of dissipative processes in gases and liquids. Novosibirsk, Nauka, 1987 (in Russian).

[25] Kurochkin V.I. A crude kinetic equation and transport equations for moderately dense gas which consists of molecules with hard core. // Teplofiz. Vys. Temp., 1990, vol. 28, No 1, p 40-46 (in Russian).

[26] Kurochkin V.I. On kinetic theory of dense gas which consists of molecules with hard core. // Zhurn. Tehn. Fiz., 1992, vol. 62, No 5, p. 13-21 (in Russian).

[27] Kurochkin V.I., Caplin S.V. Transport coefficients for dense gas which consists of molecules with hard core. // Zhurn. Tehn. Fiz., 1993, vol. 63, No 8, p. 203206 (in Russian).

[28] Kurochkin V.I., Caplin S.V. Transport coefficients for dense gas on the base of effective potential model. // Teplofiz. Vys. Temp. 1993, vol. 31, No 6, p. 903-908 (in Russian).

[29] Mulenko I.A., Khomkin A.L. The solution to Boltzmann kinetic equation for completely ionized plasma with short-range interaction between charges. // Teplofiz. Vys. Temp., 1991, vol. 29, No 6, p. 1234-1239 (in Russian).

[30] Serovskii L.A. Selfdiffusion simulation in dense gases and liquids. // Teplofiz. Vys. Temp., 1991, vol. 29, No 6, 1016-1018 (in Russian).

[31] Shmidt A.B. Short-range interaction potentials influences on thermodynamics of weakly nonideal onecomponent plasma. // Teplofiz. Vys. Temp., 1988, vol. 26, No 2, p. 387-389 (in Russian).

[32] Serovskii L.A., Vilenskaja I.E. Excess viscosity and thermal conductivity of dense gases. // Teplofiz. Vys. Temp., 1988, vol. 28, No 2, p. 610-612 (in Russian).

[33] Modinos A. Transport coefficients of a dilute gas. // Phys. Rev. A, 1992, vol. 45, No 12 , p. 8580-8582.

[34] Tokarchuk M.V., Omelyan I.P. Model kinetic equations for dense gases and liquids. // Ukr. Fiz. Zhurn., 1990, vol. 36, No 8, p. 1255-1261 (in Ukrainian).

[35] Vasserman A.A., Khasilev I.P. About effectivity taking into account a real intermolecular interaction potential in Enskog theory. // Teplofiz. Vys. Temp., 1989, vol. 27, No 1, p. 35-41 (in Russian).

[36] Vasserman A.A., Khasilev I.P. Thermal conductivity calculation for dense gas on the base of Enskog theory with taking into account a reality of intermolecular interaction potential. // Teplofiz. Vys. Temp., 1991, vol. 29, No 5, p. 878-882 (in Russian).

\footnotetext{
${ }^{1}$ Engl. transl. reads "Journal for Technical Physics"

${ }^{2}$ Engl. transl. reads "Heat Physics of High Temperatures"
} 
[37] Kincaid J.M., Perez S., Cohen E.G.D. Modified Enskog theory for fluid mixtures. // Phys. Rev. A, 1988, vol. 38, No 5, p. 3628.

[38] Alexeev B.V., Grushin I.T. The application of generalized Enskog method to calculation transport processes in mixtures of chemically reacting gases I. // Teplofiz. Vys. Temp., 1988, vol 26, No 4, p. 685-694 (in Russian).

[39] Alexeev B.V., Grushin I.T. The application of generalized Enskog method to calculation transport processes in mixtures of chemically reacting gases I. // Teplofiz. Vys. Temp., 1988, vol. 26, No 5, p. 878-887 (in Russian).

[40] Curtiss C.F. The classical Boltzmann equation of a molecular gas. // J. Chem. Phys., 1992, vol. 92, No 2, p. 1416-1419.

[41] Curtiss C.F. Bound state effects on the classical Boltzmann equation. // J. Chem. Phys., 1992, vol. 97, No 2, p. 1420-1423.

[42] Luo H., Hoheisel C. Collective transport in a molecular liquid with quadruple interaction. // Phys. Rev. A, 1991, vol. 43, No 4, p. 1819-1825.

[43] Balescu R. Statistical mechanics of charged particles. New York, Interscience Publishers, 1963.

[44] Silin V.P. Introduction to the kinetic theory of gases. Moscow, Nauka, 1971.

[45] Klimontovich Yu.L. Kinetic theory of nonideal gases and nonideal plasmas. Oxford, Pergamon Press, 1982.

[46] Chen F.F. Introduction to plasma physics and controlled fusion, vol. 1. New York, Plenum Press, 1984.

[47] Lazarev A.V., Larin A.V., Trubnikov D.N. About classical approximation of $\Omega^{(2,2)}$-integrals. // Himicheskaja Fizika ${ }^{3}$, 1992, vol. 11, No 8, p. 1034-1037 (in Russian).

[48] Trubnikov B.A. Collisions of particles in completely ionized plasma. In: Reviews of plasma physics, vol. 1 (ed. M.A.Leontovich). New York, Consultant Bureau, 1965.

[49] Braginskii S.I. Transport fenomena in plasmas. In: Reviews of plasma physics, vol. 1 (ed. M.A.Leontovich). New York, Consultant Bureau, 1965.

[50] Krylov V.I. Collision integral of kinetic equation for strongly nonideal plasma with several kinds of particles. // Fizika Plazmy ${ }^{4}$, 1991, vol. 17, No 7, p. 889-892 (in Russian).

[51] Alexeev B.V., Grushin I.T., Grushina L.P. The application of generalized Enskog method to calculation transport processes in ionized gas. // Teplofiz. Vys. Temp., 1991, vol. 29, No 2, p. 251-260 (in Russian).

[52] Alexeev B.V. On the theory of generalized Boltzmann equation. // Teplofiz. Vys. Temp., 1993, vol. 31, No 4, p. 626-635 (in Russian).

[53] Podlubny L.I., Rostovskii V.S., Filinov V.S. Theoretical investigation of conductivity of nonideal argon and xenon plasmas. // Teplofiz. Vys. Temp., 1988, vol. 26, No 26, p. 218-225 (in Russian).

[54] Maximov A.V., Chegotov M.V. On the transport theory of completely nonideal plasma. // Fizika Plazmy, 1990, vol. 16, No 5, p. 575-586 (in Russian).

[55] Nazarenko I.P., Panevin I.G. Simplified method for calculation of electric conductivity, electron conductivity and thermal diffusion in argon. // Teplofiz. Vys. Temp., 1989, vol. 27, No 3, p. 482-489 (in Russian).

[56] Rosenfeld Ya. Screening potential in strongly coupled plasmas: Reanalysis of recent highly accurate simulations. // Phys. Rev. A, 1992, vol. 46, No 2, p. 1059-1065.

[57] Klimontovich Yu.L. Statistical physics. New York, Harwood Academic Publishers, 1986.

[58] Lifshiz E.M., Pitaevskii L.P. Physical kinetics. Oxford, Pergamon Press, 1981.

[59] John W. Bond, Kenneth M. Watson, Jasper A. Welch. Atomic theory of gas dynamics. Massachusetts, Addison-Wesley Reading, 1965.

[60] Kobryn A.E., Morozov V.G., Omelyan I.P., Tokarchuk M.V. Enskog-Landau

\footnotetext{
${ }^{3}$ Engl. transl. reads "Chemical Physics"

${ }^{4}$ Engl. transl. reads "Plasma Physics"
} 
kinetic equation. Calculation ot transport coefficients for the model of charged hard spheres. // Physica A, 1996 (to be published).

[61] Kobryn A.E., Omelyan I.P. Enskog-Landau kinetic equation for twocomponent dense plasma. The solution, transport coefficients. In: Proceedings, Contributed papers of International Conference "Physics in Ukraine", volume: Plasma physics. Kiev, June 22-27, 1993, p. 135-138.

[62] Grad H. Commun. Pure \& Appl. Math. 2(1949)311.

[63] Zhdanof V.M. Transport fenomena in multicomponent plasma (Energoatomizdat, Moscow, 1982) (in Russian).

[64] Zubarev D.N., Khonkin A.D. The method for construction of normal solutions for kinetic equations with the help of boundary conditions. // Teor. Mat. Fiz., 1972, vol. 11, No 3, p. 403-412 (in Russian).

[65] Akhiezer A., Peletminskii S. Methods of statistical physics. Oxford, Pergamon, 1981.

[66] Balescu R. Transport processes in plasmas, vol. 1. Amsterdam, NorthHolland, 1988.

[67] Balabanian G.O., Khonkin A.D. The construction of generalized normal solutions for kinetic equations of gases mixture. // Teor. Mat. Fiz., 1974, vol. 18, No 1, p. 130-137 (in Russian).

[68] Zubarev D.N., Morozov V.G., Omelyan I.P., Tokarchuk M.V. Derivation of kinetic equations for the system of hard spheres using nonequilibrium statistical operator method. Preprint of Institute for Theoretical Physics, ITP-90-11R, Kiev, 1990 (in Russian).

[69] Zubarev D.N. Nonequilibrium statistical thermodynamics New York, Consultant Bureau, 1974.

[70] Tokarchuk M.V., Omelyan I.P. Normal solution of Enskog-Vlasov kinetic equation using boundary conditions method. In: Proceedings, Contributed papers of conference "Modern Problems of Statistical Physics", vol. 1. Lviv, February 3-5, 1987, p. 245-252 (in Russian).

\title{
РОЗВ'ЯЗОК КІНЕТИЧНРГО РІВНЯННЯ ЕНСКОГА-ЛАНДАУ МЕТОДОМ ГРАНИЧНИХ УМОВ
}

\author{
О.Є.Кобрин, І.П.Омелян, М.В.Токарчук
}

Розглянуто нестаціонарний нерівноважний процес, що описується кінетичним рівнянням Енскога-Ландау. Для цього рівняння знайдено нестаціонарний розв'язок у наближенні парних зіткнень та записано рівняння переносу. Вперше вклад від міжчастинкових кореляцій частинок, що взаємодіють далекосяжним потенціалом, враховано послідовно до кінця на протязі всіх обчислень. 3 врахуванням цього записано ядра переносу. Розглянуто граничні випадки: стаціонарний процес та стаціонарний процес, малі густини. Проаналізовано кінетичні коефіцієнти і нові доданки в них, що отримуються при цьому. Використаний метод придатний до гідродинамічного опису швидких процесів. 